\title{
COUNTABLE HOMOGENEOUS TOURNAMENTS
}

BY

\author{
A. H. LACHLAN
}

\begin{abstract}
A tournament $T$ is called homogeneous just in case every isomorphism of subtournaments of smaller cardinality can be lifted to an automorphism of $T$. It is shown that there are precisely three homogeneous tournaments of power $\boldsymbol{\aleph}_{0}$. Some analogous results for 2-tournaments are obtained.
\end{abstract}

In this paper we characterize countable tournaments which are homogeneous in the sense of Fraissé [1]. As far as we know, interest in such a characterization dates from 1976 when Woodrow showed in his dissertation [10] that the only finite homogeneous tournaments have orders 1 and 3 and that up to isomorphism there are only two countably infinite homogeneous tournaments which do not embed the tournament $D$ shown in Figure 1. The next year Woodrow and the author [7] characterized countable homogeneous graphs. This reawakened the author's interest in the tournament problem. Independently, Schmerl [9] characterized countable homogeneous partial orderings and also formulated the question as to which countable tournaments are homogeneous.

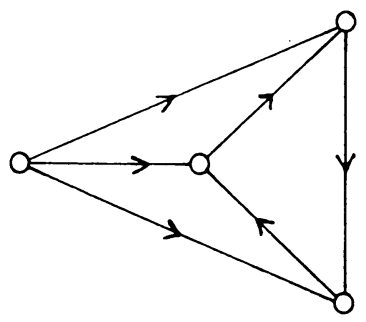

FIGURE 1

Apart from that of Woodrow mentioned above, the only work relevant to the problem that we know of is the unpublished proof of $R$. Morrow that any homogeneous tournament embedding $D$ must also embed every tournament of order 6 . Here we shall show that a homogeneous tournament embedding $D$ embeds every finite tournament.

Our proof requires labeled tournaments. For $n<\omega$ an $n$-tournament is a tournament in which each vertex is labeled by exactly one of the natural numbers $<n$. As a by-product of our characterization of countable homogeneous tournaments we also find a corresponding characterization for 2 -tournaments. The problem for $n$-tournaments $(n>2)$ is open.

Received by the editors August 20, 1980 and, in revised form, January 18, 1983.

1980 Mathematics Subject Classification. Primary 02H05, 05C 20. 
The plan of the paper is as follows. In $§ 1$ we develop notation, terminology, and prove several propositions required later on. In $\$ 2$ we give Woodrow's characterization of countable homogeneous tournaments omitting $D$ and an analogous result for 2-tournaments. In $\$ 3$, omitting the proofs of some lemmas, we prove the main theorem which says that apart form those found by Woodrow there is just one countable homogeneous tournament up to isomorphism. In $\$ \S 4$ and 5 the propositions, required to complete the proof given in $\$ 3$, are developed. Finally, in $\$ 6$ we characterize countable homogeneous 2-tournaments.

One word of warning: The next section consists mostly of definitions and notations. The reader is advised to read it quickly and then to use it for reference while working through the rest of the paper. The summary at the end of $\S 1$ will assist in this regard.

I would like to record my thanks to Ron Morrow and Robert Woodrow for the discussions we had when the conjecture proved here first came to our notice. My thanks also to Calvin Foote who helped me revise the paper.

1. Preliminaries. A tournament $T$ is a structure $\left\langle|T|, E_{T}\right\rangle$, where $|T|$ is a nonempty set, the universe or vertex set of $T$, and $E_{T}$ is a binary relation on $T$ such that

$$
T \vDash \forall x \forall y[x E y \rightarrow(x \neq y \& y E x) . \& . x \neq y \rightarrow(x E y \vee y E x)] .
$$

We call $E_{T}$ the dominance relation of $T$ and if $a E_{T} b$ we say that $a$ dominates $b$. In diagrams of tournaments the vertices are denoted by points, and if $a$ dominates $b$ there will be an edge directed from the point representing $a$ to the point representing $b$.

The tournament $T$ is vertex transitive if for all $a, b \in T$ there exists $\alpha \in$ aut $T$, the automorphism group of $T$, such that $\alpha(a)=b$.

For $1<n<\omega$, an n-tournament $\mathbf{T}$ is a structure $\left\langle|\mathbf{T}|, E_{\mathrm{T}}, R_{\mathrm{T}}^{0}, \ldots, R_{\mathrm{T}}^{n-1}\right\rangle$ such that $\left\langle|\mathrm{T}|, E_{\mathbf{T}}\right\rangle$ is a tournament, and $R_{\mathrm{T}}^{0}, \ldots, R_{\mathrm{T}}^{n-1}$ are pairwise disjoint unary relations on $|\mathbf{T}|$ whose union is $|\mathbf{T}|$. The vertices in $R_{\mathrm{T}}^{i}$ are to be thought of as being labeled by $i$.

We call $T$ (or T) transitive, not to be confused with "vertex transitive", if $E_{T}$ (or $\left.E_{\mathrm{T}}\right)$ is transitive.

We use $T, U, V, \ldots$ to denote tournaments and $\mathbf{T}, \mathbf{U}, \mathbf{V}, \ldots$ to denote 2-tournaments. For an indexed family of tournaments (2-tournaments) we use $T\{j\}(\mathbf{T}\{j\})$ to denote the member of the family with index $j$.

The class of all tournaments (2-tournaments) is denoted by $\mathcal{T}(\mathcal{T})$. The class of all finite tournaments (2-tournaments) is denoted by $\mathscr{F}(\mathscr{F})$. By convention, all tournaments and 2-tournaments considered below are countable.

We write $T \approx U$ to mean that $T$ and $U$ are isomorphic. Our notions of embedding and substructure (i.e., subtournament) are the usual ones from first-order model theory. For structures $T, U$, by $T \subset U$ we mean that $T$ is a substructure of $U$. For sets $A, B$, by $A \subset B$ we mean that $A$ is a subset of $B$. We say that $T$ omits $U$ if $T$ does not embed $U$. The cardinality of a set $A$ is denoted $|A|$. The order or cardinality of a tournament $T$ is $\|T\|$; similarly, for $n$-tournaments. In other cases where there is an obvious extension of a notion, definition, or notation to $n$-tournaments we may simply assume it without special mention. We shall write $a \in T$ instead of $a \in|T|$. 
If $T$ is finite and $a \in T$ then the score pair of $a$ is $(m, n)$, where $m$ is the number of vertices $a$ dominates and $n$ is the number dominating $a$. Clearly, $m+n+1=\|T\|$.

After Fraissé [1] a tournament $T$ is homogeneous if every isomorphism between finite substructures of $T$ may be extended to an automorphism of $T$. The class of homogeneous tournaments (2-tournaments) is denoted by $\mathcal{H}(\mathcal{H})$.

A class $\mathscr{Q} \subset \mathscr{F}$ is called an amalgamation class if $\mathscr{Q}$ is closed under isomorphism and subtournaments, and for all $T, U \in \mathbb{Q}$, agreeing on $|T| \cap|U|$, there exist $V \in \mathbb{Q}$ and embeddings of $T, U$ in $V$ which agree on $|T| \cap|U| . \mathscr{Q}$ is a strong amalgamation class if, in addition, for all $T, U \in A$ agreeing on $|T| \cap|U|$, there exists $V \in A$ extending both $T$ and $U$. Define

$$
\mathscr{Q}(T)=\{U \in \mathscr{F}: T \text { embeds } U\} .
$$

Fraissé [1] proved a general result which for tournaments may be stated:

1.1. (1) If $T \in \mathcal{H}$, then $\mathscr{Q}(T)$ is an amalgamation class.

(2) If $\mathcal{C} \subset \mathcal{F}$ is an amalgamation class there exists $T \in \mathcal{H}$ unique up to isomorphism such that $\mathbb{Q}(T)=\bigodot$.

This theorem is the key to our whole investigation, because we find the possibilities for $T \in \mathcal{H}$ by discovering all amalgamation classes of tournaments.

Working from left to right the tournaments depicted in Figure 2 will be denoted $C, L^{3}$ and $J$. For $1<n<\omega, L^{n}$ denotes transitive tournament of order $n$. The tournament of order 1 is denoted $I$. In diagrams of 2-tournaments the vertices will appear in two boxes; those in the left-hand box are labeled 0 and those in the right-hand box are labeled 1. The 2-tournaments shown in Figure 3 are denoted $\mathbf{F}$ and $\mathbf{G}$, respectively. Let $Q$ denote the tournament such that $|Q|$ is the set of rational numbers and $E_{Q}$ is the strict ordering of $|Q|$. Let $\mathbf{Q}$ be an expansion of $Q$ to a 2-tournament such that both the 0-labeled and the 1-labeled vertices are dense in $Q$. The back-and-forth argument, which shows that a countable dense linear ordering is unique up to isomorphism, easily extends to show that $\mathbf{Q}$ is unique up to isomorphism and that both $Q$ and $\mathbf{Q}$ are homogeneous.
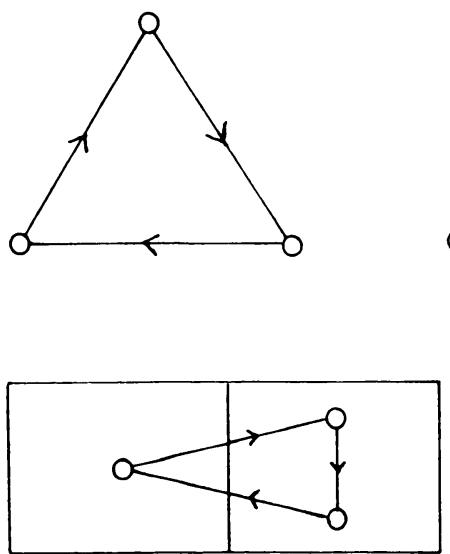
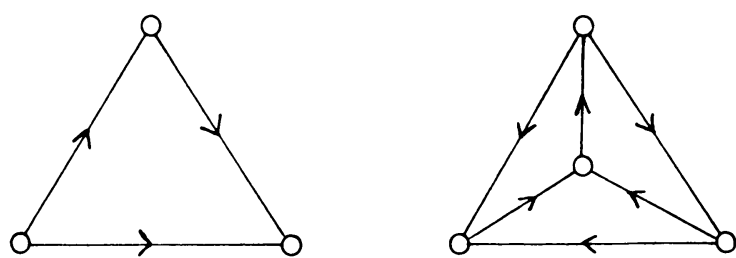

Figure 2

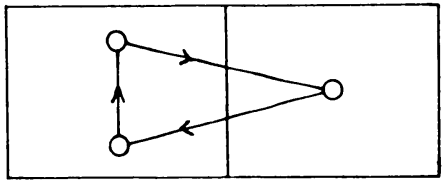

Figure 3 
Let $T$ be a tournament of order $>1$ and $t \in T$. Then $(T, t)$ denotes the 2-tournament obtained from $T$ by deleting $t$ and labeling $u \in|T|-\{t\}$ by 0 or 1 according as $t$ dominates or is dominated by $u$. Conversely, if $\mathbf{T}$ is a 2-tournament, then (T) denotes the tournament obtained from $\mathbf{T}$ by deleting the labels and adjoining a new vertex which dominates the vertices which were labeled 0 in $\mathbf{T}$ and is dominated by those which were labeled 1 . Let $\mathbf{T}$ be a 2 -tournament, then $\mathbf{T} \leftrightarrow$ denotes the 2-tournament obtained from $\mathbf{T}$ by reversing the dominance relation between vertices with different labels.

Let $P$ be the tournament such that $|P|=|Q|$ and

$$
p_{0} E_{P} p_{1} \equiv \exists n\left[0<p_{1}-p_{0} \pm 2 n \pi<\pi\right] \text {. }
$$

A neighbourhood in $P$ is a subset of $|P|$ of the form

$$
\left\{p \in|P|: p_{0} E_{P} p \& p E_{P} p_{1}\right\}
$$

where $p_{0} E_{P} p_{1}$; similarly for $Q$. A set $A \subset|P|$ is called dense in $P$ if it intersects every neighbourhood in $P$; similarly for $Q$. One can conveniently think of $P$ as consisting of the points on the unit circle whose polar coordinates are rational, and where $\left(1, p_{0}\right)$ dominates $\left(1, p_{1}\right)$ if the shortest path along the circumference of the unit circle from $\left(1, p_{0}\right)$ to $\left(1, p_{1}\right)$ is transcribed anticlockwise. Let + denote the usual addition operation on the rationals. For $a, b \in P$ the mapping $p \mapsto p+b-a$ ( $p \in P$ ) is an automorphism of $P$ taking $a$ to $b$. Thus $P$ is vertex transitive. Let $\mathbf{U}$ denote $(P, q)^{\hookrightarrow}$ where $q \in P$. We claim that $\mathbf{U}$ is a copy of $\mathbf{Q}$. To see this it is enough to show that $\mathbf{U}$ is transitive and that $\left|\mathbf{U}_{i}\right|$ is dense in $\mathbf{U}(i<2)$. For transitivity suppose $a E_{\mathbf{U}} b$ and $b E_{\mathbf{U}} c$. Then four cases arise:

(i) $a, b, c \in \mathbf{U}_{i}$;

(ii) $a, b \in \mathbf{U}_{i}$ and $c \in \mathbf{U}_{1-i}$;

(iii) $a \in \mathbf{U}_{i}$ and $b, c \in \mathbf{U}_{1-i}$;

(iv) $a, c \in \mathbf{U}_{i}$ and $b \in \mathbf{U}_{1-i}$.

Consider, for example, case (ii) with $i=0$. We have $a E_{P} b$ and $c E_{P} b$, and so in $P$ the points are arranged as shown in Figure 4. It is clear that $c E_{P} a$, whence $a E_{\mathbf{U}} c$ as required. The other cases are equally easy. To show density suppose $a E_{\mathrm{U}} b$. We must find $c \in \mathbf{U}_{i}$ such that $a E_{\mathbf{U}} c$ and $c E_{\mathbf{U}} b$. If $b \in \mathbf{U}_{i}$, take $c=b-\varepsilon$ for any sufficiently small positive rational $\varepsilon$. If $b \in \mathbf{U}_{1-i}$, take $c=b+\varepsilon$ for any rational $\varepsilon<\pi$ such that $\pi-\varepsilon$ is sufficiently small.

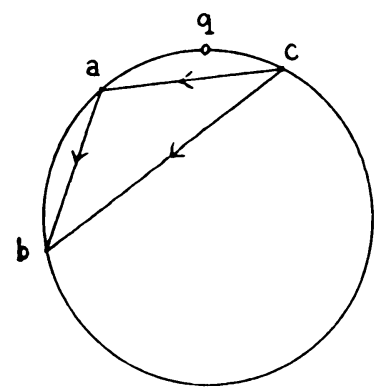

FigURE 4 
We now establish the homogeneity of $P$. Let $\sigma: A \rightarrow B$ be an isomorphism between subtournaments $A$ and $B$ of $P$. Choose $a \in A$. By vertex transitivity there is an $\alpha \in$ aut $P$ such that $\alpha(a)=\sigma(a)$. Then $\alpha(A)$ and $B$ are isomorphic via $\sigma \alpha^{-1}$ which fixes $b=\sigma(a)$. If $\beta \in$ aut $P$ extends $\sigma \alpha^{-1}$, then $\beta \alpha \in$ aut $P$ extends $\sigma \alpha^{-1} \alpha=\sigma$. We may, therefore, assume that there exists $q \in A \cap B$ such that $\sigma(q)=q$. Let $A^{\prime}$, $B^{\prime}$ be the subtournaments of $(P, q)^{\hookrightarrow}$ with universes $A-\{q\}, B-\{q\}$. Then $\sigma\left\lceil(A-\{q\})\right.$ is an isomorphism of $A^{\prime}$ onto $B^{\prime}$. Now $(P, q)^{\leftrightarrow}$, being a copy of $\mathbf{Q}$, is homogeneous. Let $\gamma \in \operatorname{aut}(P, q)^{\leftrightarrow}$ extend $\sigma \uparrow(A-\{q\})$. Then $\gamma \cup\{\langle q, q\rangle\}$ is the required automorphism of $P$ extending $\sigma$.

1.2. Let $q_{i} \in Q(i<n)$ be distinct. There exist neighbourhoods $A_{i}(i<n)$ such that $q_{i} \in A_{i}(i<n)$ and if $a_{i} \in A_{i}(i<n)$ then $q_{i} \mapsto a_{i}(i<n)$ is an isomorphism of subtournaments; similarly for $P$.

Now form $\mathbf{P}$ from $P$ by partitioning $|P|$ so that both the 0 -labeled vertices and the 1-labeled vertices are dense. From 1.2 we see that this determines $\mathbf{P}$ uniquely up to isomorphism and that $\mathbf{P} \in \mathcal{H}$.

By a cut in $Q$ we mean $A \subset|Q|$ such that $\varnothing \neq A \neq|Q|, A$ has no greatest element, and $A$ is closed downwards. By a cut in $P$ we mean $A \subset|P|$ satisfying

(i) if $a, b \in A$ and $a E_{P} b$ then $c \in A$ for all $c$ such that $a E_{P} c$ and $c E_{P} b$, and

(ii) if $a \in P$ then there exist $b \in A$ and $c \notin A$ such that $a E_{P} b$ and $a E_{P} c$.

Let $R$ be a countable homogeneous tournament such that $\mathcal{Q}(R)=\mathscr{F}$, and $\mathbf{R}$ be a countable homogeneous tournament such that $\mathcal{Q}(R)=\mathcal{F}$. From $1.1 R$ and $\mathbf{R}$ are unique up to isomorphism.

For $i<2$ let $\mathbf{I}^{i}, \mathbf{C}^{i}, \mathbf{D}^{i}, \mathbf{P}^{i}, \mathbf{Q}^{i}, \mathbf{R}^{i}$ and $\mathbf{L}^{n, i}$ be $i$-labeled copies of $I, C, D, P, Q, R$ and $L^{n}$, respectively. If $i_{0}, i_{1}, \ldots, i_{k} \in\{0,1\}$ then $\mathbf{E}\left(i_{0} i_{1} \cdots i_{k}\right)$ denotes the 2-tournament with universe $\{0,1, \ldots, k\}$ in which each vertex dominates those less than it, and $j$ is $i$-labeled if and only if $i_{j}=i$. Clearly every finite transitive 2-tournament is isomorphic to one of the forms $\mathbf{E}\left(i_{0} i_{1} \cdots i_{k}\right)$. The class of all finite transitive tournaments (2-tournaments) will be denoted by $\mathcal{L}(\mathfrak{L})$.

If $T$ is a tournament, then $\bar{T}$ denotes the complement of $T$ obtained by reversing the dominance relation in $T$, and $T(*)$ denotes the tournament obtained by adjoining a new vertex which dominates all the others. If $t \in T$ then $T[t \rightarrow]$ denotes the restriction of $T$ to the vertices dominated by $t$, and $T[\rightarrow t]$ the restriction of $T$ to the vertices which dominate $t$.

If $\mathbf{T}$ is a 2-tournament and $i<2$, then $\mathbf{T}_{i}$ denotes the restriction of $\mathbf{T}$ to the $i$-labeled vertices. If $t \in \mathbf{T}_{i}$, then $(\mathbf{T} ; t)$ denotes the 2-tournament $\mathbf{U}$ defined by $\left|\mathbf{U}_{0}\right|=\left|\mathbf{T}_{1-i}[t \rightarrow]\right|,\left|\mathbf{U}_{1}\right|=\mathbf{T}_{1-i}[\rightarrow t]$, and $E_{\mathbf{U}}=E_{\mathbf{T}}|| \mathbf{U} \mid$. If the isomorphism type of ( $\mathbf{T}: t)$ for $t \in \mathbf{T}_{1-i}$ is independent of $t$, then ( $\left.\mathbf{T}: i\right)$ denotes a 2-tournament having that common isomorphism type. By $F(\mathbf{T})$ we denote the tournament obtained from $\mathbf{T}$ by deleting the labels on the vertices. For $i<2, \mathbf{T}(i)$ denotes the 2-tournament obtained from $\mathbf{T}$ by adjoining a new $i$-labeled vertex which dominates all the other vertices. $\overline{\mathbf{T}}$ denotes the complement of $\mathbf{T}$ obtained by reversing the dominance relation. By the dual of $\mathbf{T}$ we mean the 2-tournament $\tilde{\mathbf{T}}$ obtained from $\mathbf{T}$ by switching the labels 0 and 1 . The 2-tournaments $\mathbf{T}^{\leftrightarrow}, \overline{\mathbf{T}}$ and $\tilde{\mathbf{T}}$ are examples of variants of $\mathbf{T}$. 
More generally, $\mathbf{U}$ is a variant of $\mathbf{T}$ if it can be obtained by a sequence of operations of the following kinds: switching the labels 0 and 1 , reversing the dominance relation between 0-labeled vertices, reversing the dominance relation between 1-labeled vertices, reversing the dominance relation between vertices with unlike labels. Including $\mathbf{T}$ itself there are a possible 16 variants of $\mathbf{T}$. We say that $\mathbf{U}$ is $\mathbf{T}$ up to a variant if $\mathbf{U}$ is isomorphic to one of the variants of $\mathbf{T}$.

The wreath product $T[U]$ is defined by: $|T[U]|=|T| \times|U|$ and

$$
\left(t_{0}, u_{0}\right) E_{T[U]}\left(t_{1}, u_{1}\right) \equiv\left[t_{0} E_{T} t_{1} \text { or }\left(t_{0}=t_{1} \& u_{0} E_{U} u_{1}\right)\right] \text {. }
$$

Intuitively, $T[U]$ is obtained by replacing each vertex of $T$ by a copy of $U$. We define $T[\mathbf{U}]$ and $\mathbf{T}[U]$ similarly, where each vertex in the composition takes the label of its labeled component.

Let $Q \subset \mathscr{F}$. We say $T$ embeds $\mathcal{Q}$ if $T$ embeds every member of $\mathcal{Q}$. If $\mathcal{Q}, \mathscr{G} \subset \mathscr{F}$, we say that $\mathcal{Q}$ implies $\mathscr{B}$, written $\mathcal{Q} \Rightarrow \mathscr{G}$, if every $T \in \mathcal{H}$ embedding $\mathcal{Q}$ also embeds $\mathscr{B}$. For finite classes we often simply list their members. Thus $\mathcal{Q} \Rightarrow U$ means $\mathcal{Q} \Rightarrow\{U\}$, and $U, V \Rightarrow \mathbb{Q}$ means $\{U, V\} \Rightarrow \mathbb{Q}$, etc. These definitions and conventions carry over to 2-tournaments. Implications between subsets of $\mathscr{F}$ and $\mathscr{F}$ are also defined. If $\mathcal{Q} \subset \mathscr{F}$ and $\mathscr{B} \subset \mathscr{F}$, then $\mathcal{Q} \Rightarrow \mathscr{B}$ means that $\mathcal{Q} \Rightarrow\{(\mathbf{T})$ : $\mathbf{T} \in \mathscr{B}\}$, while $\mathscr{B} \Rightarrow \mathbb{Q}$. means that for every $U \in \mathbb{Q}$ there exists $\mathbf{T} \in \mathscr{F}$ such that $\mathscr{B} \Rightarrow \mathbf{T}$ and $(\mathbf{T})$ embeds $U$. It is easy to see that $\Rightarrow$ is transitive except in the following cases: If $\mathcal{Q} \Rightarrow \mathscr{B}$ and $\mathscr{B} \Rightarrow \mathcal{C}$ or $\mathscr{B} \Rightarrow \mathcal{C}$, then it is not generally true that $\mathbb{Q} \Leftrightarrow \mathcal{C}$ or $\mathbb{Q} \Rightarrow \mathcal{C}$, respectively.

Let $(\mathbb{Q})$ denote $\{(\mathbf{T}): \mathbf{T} \in \mathbb{Q}\}$. Part of the transitivity of $\Rightarrow$ lies in the observation that, if $\mathbb{Q} \Rightarrow \mathscr{B}$, then $(\mathbb{Q}) \Rightarrow(\mathscr{B})$. We will use this several times below.

As an example of implication we have

1.3. (1) $C, L^{3} \Rightarrow J$.

(2) $\mathbf{E}(01), \mathbf{E}(00) \Rightarrow \mathbf{E}(001)$.

Proof. (1) Let $T \in \mathfrak{T C}$ embed $C$ and $L^{3}$. From 1.1 $\mathscr{Q}(T)$ is an amalgamation class whence $T$ embeds the tournament $U$ shown in Figure 5 which is obtained by amalgamating $L^{3}$ and $C$ over a common edge. Whichever way the broken edge is directed, $U \approx J$. In diagrams of amalgamations the edges of the tournament or 2-tournament over which we are amalgamating will be drawn more heavily.

(2) Let $\mathbf{T} \in \mathcal{H}$ embed $\mathbf{E}(01)$ and $\mathbf{E}(00)$, and for a contradiction argument suppose $\mathbf{T}$ does not embed $\mathbf{E}(001)$. Let $a \in \mathbf{T}_{1}$. Observe that then there is a unique $b \in \mathbf{T}_{0}$ dominated by $a$ (see Figure 5). Since $\mathbf{T}$ embeds $\mathbf{E}(00)$ there are $c$ and $d$ in $\mathbf{T}_{0}$ dominated by and dominating $b$, respectively. Since $\mathbf{T}$ does not embed $\mathbf{E}(001), c$ and $d$ both dominate $a$. Now there must be an automorphism $\alpha$ of T fixing $a$ and taking $c$ into $d$. Clearly, $\alpha(b) \neq b$ and yet $a$ dominates $\alpha(b)$. This is the desired contradiction.

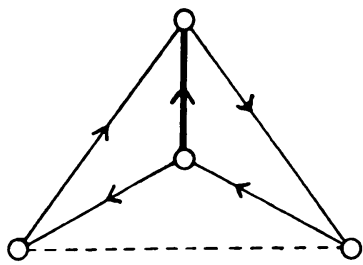

U

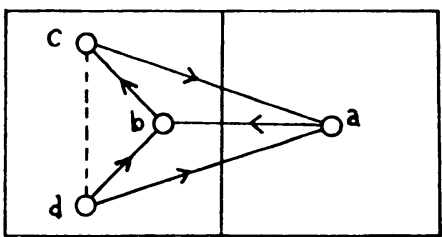

T

FIGURE 5 
If $\mathscr{Q} \subset \mathscr{B} \subset \mathscr{F}$ and $\mathscr{Q} \Rightarrow \mathscr{B}$ then we say that $\mathscr{Q}$ is a base for $\mathscr{R}$. If $T \Rightarrow U$ and $U \Rightarrow T$ then $T$ and $U$ are called equivalent.

From Ramsey's Theorem it follows that for every $n$ there exists a least number $r(n)$ such that every $T \in \mathcal{T}$ of order $\geqslant r(n)$ embeds $L^{n}$. Two of the consequences of Ramsey's Theorem which we need are:

1.4. (1) If $T \in \mathcal{H}$ embeds every $U \in \mathscr{F}$ which can be partitioned into a singleton and a transitive tournament, then $T$ embeds every tournament which can be partitioned into two transitive tournaments.

(2) If $\mathbf{T} \in \mathcal{H}$ embeds every $\mathbf{U}$ which can be partitioned into a 0 -labeled singleton and a 1-labeled transitive tournament, then $\mathbf{T}$ embeds every $\mathbf{U}$ which can be partitioned into a 0-labeled transitive tournament and a 1-labeled transitive tournament.

Proof. We shall only prove (1) because it will be clear that exactly the same method also works for (2). Let $T \in \mathcal{H}$ embed any $U \in \mathscr{F}$ which can be partitioned into a singleton and a transitive tournament. Let $V \in \mathscr{F}$ and $|V|=|X| \dot{U}|Y|$, where $\dot{U}$ denotes disjoint union, and $X$ and $Y$ are transitive subtournaments of $V$. We must show that $T$ embeds $V$.

Let $\|X\|=n<\omega$. Let $\gamma_{0}, \ldots, \gamma_{m-1}$ be an enumeration without repetitions of all one-one mappings from $|X|$ into $r(n)$. Let $p=m+r(n)$ and $a \notin|Y| \times p$. For each $i<r(n)$ let $V\{i\}$ be the tournament defined by

$$
|V\{i\}|=\{a\} \cup(|Y| \times p), \quad V\{i\} \uparrow(|Y| \times p)=Y\left[L^{p}\right]
$$

and

$a$ dominates $(y, j)$ in $V\{i\} \equiv[(m \leqslant j<p \& j=m+i)$ or

$$
\left.\left(j<m \& i \in \operatorname{rng} \gamma_{j} \& \gamma_{j}^{-1}(i) \text { dominates } y \text { in } V\right)\right] \text {. }
$$

Without loss of generality, suppose that $Y\left[L^{p}\right]$ is a subtournament of $T$. Notice that $V\{i\}$ is the union of $Y\left[L^{p}\right]$ and a singleton. For each $i<r(n)$ choose $t_{i} \in T$ such that the mapping which takes $a$ to $t_{i}$ and is the identity on $Y\left[L^{p}\right]$, embeds $V\{i\}$ in $T$. The vertices $t_{i}(i<r(n))$ are distinct because $a$ dominates $(y, m+i)$ in $V\{i\}$ but not in $V\{j\}$ for any $j \neq i$. By choice of $r(n)$ there exists $j<m$ such that $x \mapsto t_{\gamma_{j}(x)}(x \in X)$ is an embedding of $X$ in $T$. Let $x \in X$ and $y \in Y$. By choice of $t_{\gamma_{j}(x)}$,

$$
t_{\gamma_{j}(x)} \text { dominates }(y, j) \text { in } T \equiv a \text { dominates }(y, j) \text { in } V\left\{\gamma_{j}(x)\right\}
$$

and by definition of $V\left\{\gamma_{j}(x)\right\}$

$$
a \text { dominates }(y, j) \text { in } V\left\{\gamma_{j}(x)\right\} \equiv \gamma_{j}^{-1} \gamma_{j}(x) \text { dominates } y \text { in } V \text {. }
$$

Combining the last two equivalences

$$
t_{\gamma_{j}(x)} \text { dominates }(y, j) \text { in } T \equiv x \text { dominates } y \text { in } V \text {. }
$$

This shows that the map $x \mapsto t_{\gamma_{f}(x)}(x \in X)$ and $y \mapsto(y, j)(y \in Y)$ embeds $V$ in $T$ as required.

We must discuss certain operations on tournaments and 2-tournaments which will be useful in the sequel. 
Letting $|C|=\{x, y, z\}$ and $E_{C}=\{(x, y),(y, z),(z, x)\}$ we define $C[\mathbf{X}, \mathbf{Y}, \mathbf{Z}]$ by

$$
|C[\mathbf{X}, \mathbf{Y}, \mathbf{Z}]|=(\{x\} \times|\mathbf{X}|) \cup(\{y\} \times|\mathbf{Y}|) \cup(\{z\} \times|\mathbf{Z}|),
$$

$(a, b)$ dominates $(c, d)$ if and only if $a$ dominates $c$ or $a=c$ and $b$ dominates $d$, and $(a, b)$ is $i$-labeled if and only if $b$ is $i$-labeled. We define $D[\mathbf{X}, \mathbf{Y}, \mathbf{Z}, \mathbf{W}]$ similarly, where $|D|=\{x, y, z, w\}$ and $E_{D}=\{(x, y),(x, z),(x, w),(y, z),(z, w),(w, y)\}$.

By $[T, U]$ we denote the tournament obtained by first replacing $U$ by a copy disjoint from $T$, if necessary, and then setting $|[T, U]|=|T| \cup|U|$ and

$$
E_{[T, U]}=E_{T} \cup E_{U} \cup(|T| \times|U|) .
$$

The operations described so far are called replacement operations because the value is obtained by replacing some vertices of a fixed tournament or 2-tournament by arguments of the operation. An earlier example was the operation $\mathbf{T} \mapsto \mathbf{T}(i)$ for each $i<2$. However, in formulating the notion of replacement operation we make the restriction that each argument replaces at most one vertex of the fixed tournament. Thus, while $\langle\mathbf{T}, \mathbf{U}\rangle \mapsto[\mathbf{T}, \mathbf{U}]$ is a binary replacement operation, the fixed tournament being $L^{2}$, the unary operation $\mathbf{T} \mapsto[\mathbf{T}, \mathbf{T}]$ is not a replacement operation.

We now define some more complex operations on 2-tournaments which will play a role only in $\S 5$. Firstly, $\langle\langle\mathbf{E}(01), \mathbf{T}\rangle\rangle$ is obtained from $\mathbf{T}$ by adjoining a copy $\mathbf{U}$ of $\mathbf{E}(01)$ and letting each $u \in \mathbf{U}$ dominate $\mathbf{T}_{0}$ and be dominated by $\mathbf{T}_{1}$. The 2-tournament $\langle\langle\mathbf{E}(10), \mathbf{T}\rangle\rangle$ is defined similarly. For $i\left\langle 2,\left\langle\left\langle\mathbf{C}^{i}, \mathbf{T}\right\rangle\right\rangle\right.$ is the 2-tournament $\mathbf{U}$ defined by letting $V$ be a copy of $C$ disjoint from $\mathbf{T}, v \in V,\left|\mathbf{U}_{i}\right|=|V|,\left|\mathbf{U}_{1-i}\right|=|\mathbf{T}|$, and

$$
E_{\mathbf{U}}=E_{V} \cup E_{\mathbf{T}} \cup\left(|V| \times\left|\mathbf{T}_{0}\right|\right) \cup\left((|V|-\{v\}) \times\left|\mathbf{T}_{1}\right|\right) \cup\left(\left|\mathbf{T}_{1}\right| \times\{v\}\right) .
$$

We define a kind of inverse of this last operation. Let $\mathbf{U} \in \mathcal{H}$ embed $\mathbf{C}^{i}$. Choose a copy $\mathbf{V}$ of $\mathbf{C}^{i}$ in $\mathbf{U}$ and let $v \in \mathbf{V}$ then $\left\langle\left\langle\mathbf{C}^{i}, \mathbf{U}\right\rangle\right\rangle^{-1}$ denotes the tournament $\mathbf{T}$ defined by

$$
\begin{aligned}
& \left|\mathbf{T}_{0}\right|=\left\{u \in \mathbf{U}_{1-i}: \text { each } w \in V \text { dominates } u\right\}, \\
& \left|\mathbf{T}_{1}\right|=\left\{u \in \mathbf{U}_{1-i}: \text { each } w \in V-\{v\} \text { dominates } u\right\}-\left|\mathbf{T}_{0}\right|
\end{aligned}
$$

and

$$
E_{\mathbf{T}}=E_{\mathbf{U}}|| \mathbf{T} \mid .
$$

If $\mathbf{U} \in \mathcal{H}$ embeds $\left\langle\left\langle\mathbf{C}^{i}, \mathbf{T}\right\rangle\right\rangle$, then $\left\langle\left\langle\mathbf{C}^{i}, \mathbf{U}\right\rangle\right\rangle^{-1}$ is a homogeneous tournament embed$\operatorname{ding} \mathbf{T}$.

For $i<2,\left\langle\left\langle\mathbf{D}^{i}, \mathbf{T}\right\rangle\right\rangle$ is the 2-tournament $\mathbf{U}$ defined by letting $V$ be a copy of $D$ disjoint from $\mathbf{T},\left|\mathbf{U}_{i}\right|=|V|,\left|\mathbf{U}_{1-i}\right|=|\mathbf{T}|$, and

$$
E_{\mathbf{U}}=E_{V} \cup E_{\mathbf{T}} \cup\left(|V| \times\left|\mathbf{T}_{0}\right|\right) \cup\left(\left|\mathbf{T}_{1}\right| \times|V|\right) .
$$

Finally, for $i<2,\left(\mathbf{C}^{i}, \mathbf{T}\right)$ is the 2-tournament $\mathbf{U}$ defined as follows. Let $V$ be a copy of $C$ disjoint from T, where $|V|=\{x, y, z\}$ and $E_{V}=\{(x, y),(y, z),(z, x)\}$. We set $\left|\mathbf{U}_{i}\right|=|V|,\left|\mathbf{U}_{1-i}\right|=|\mathbf{T}|$, and

$$
\begin{aligned}
E_{\mathbf{U}}= & E_{V} \cup E_{\mathbf{T}} \cup\left(\left|\mathbf{T}_{0}\right| \times\{x, z\}\right) \cup\left(\{y\} \times\left|\mathbf{T}_{0}\right|\right) \\
& \cup\left(\left|\mathbf{T}_{1}\right| \times\{x, y\}\right) \cup\left(\{z\} \times\left|\mathbf{T}_{1}\right|\right) .
\end{aligned}
$$


An important property of these operations is

1.5. (1) Let $\boldsymbol{\Phi}: \mathcal{F} \rightarrow \mathscr{F}$ be a replacement operation or any of the mappings

$$
\mathbf{T} \mapsto\langle\langle\mathbf{E}(01), \mathbf{T}\rangle\rangle, \mathbf{T} \mapsto\left\langle\left\langle\mathbf{C}_{i}, \mathbf{T}\right\rangle\right\rangle, \mathbf{T} \mapsto\left\langle\left\langle\mathbf{D}_{i}, \mathbf{T}\right\rangle\right\rangle, \mathbf{T} \mapsto\left(\mathbf{C}_{i}, \mathbf{T}\right)
$$

just described. If $\mathbf{T}\{0\}, \mathbf{T}\{1\}, \ldots, \mathbf{T}\{m\} \Rightarrow \mathbf{T}$, then $\boldsymbol{\Phi}(\mathbf{T}\{0\}), \ldots, \boldsymbol{\Phi}(\mathbf{T}\{m\}) \Rightarrow \boldsymbol{\Phi}(\mathbf{T})$.

(2) Let $\boldsymbol{\Psi}$ be an $n$-ary operation on $\mathcal{F}$ which is either a replacement operation or one of the other operations listed in (1). If $\mathbb{Q} \subset \mathcal{F}$ and $\mathbb{Q} \Rightarrow \boldsymbol{\Psi}(\mathbf{T}\{0\}, \ldots, \mathbf{T}\{n\})$ for all $\mathbf{T}\{0\}, \ldots, \mathbf{T}\{n\} \in \mathbb{Q}$, then $\mathbb{Q} \Rightarrow \boldsymbol{\Psi}(\mathbf{T}\{0\}, \ldots, \mathbf{T}\{n\})$ for all $\mathbf{T}\{0\}, \ldots, \mathbf{T}\{n\}$ such that $\mathbb{Q} \Rightarrow \mathbf{T}\{0\}, \ldots, \mathbf{T}\{n\}$.

Proof. (1) Let $\mathbf{T}\{0\}, \ldots, \mathbf{T}\{m\} \Rightarrow \mathbf{T}$ and $\boldsymbol{\Phi}: \mathscr{F} \rightarrow \mathscr{F}$ be an operation of the kind specified. Let $\mathbf{U} \in \mathcal{H}$ embed $\boldsymbol{\Phi}(\mathbf{T}\{0\}), \ldots, \boldsymbol{\Phi}(\mathbf{T}\{m\})$. We have to show that $\mathbf{U}$ embeds $\boldsymbol{\Phi}(\mathbf{T})$. The key observation is that

$$
\mathcal{C}=\{\mathbf{V} \in \mathscr{F}: \mathbf{U} \text { embeds } \boldsymbol{\Phi}(\mathbf{V})\}
$$

is an amalgamation class. Consider the operation $\mathbf{T} \mapsto \mathbf{T}(0)$ as an example. Fix any $u \in \mathbf{U}_{0}$, then $\mathcal{C}=\mathbb{Q}(\mathbf{U}[u \rightarrow])$. The homogeneity of $\mathbf{U}$ guarantees that $\mathbf{U}[u \rightarrow]$ is homogeneous. Hence $\mathcal{C}$ is an amalgamation class by 1.1(1). By assumption, $\mathbf{T}\{0\}, \ldots, \mathbf{T}\{m\} \in \mathcal{C}$, and by $1.1(2)$ it follows that $\mathbf{T} \in \mathcal{C}$ since $\mathbf{T}\{0\}, \ldots, \mathbf{T}\{m\} \Rightarrow \mathcal{C}$. This is sufficient.

(2) Notice that if $\Psi$ is unary then the result is immediate from (1). If $\Psi$ is $n$-ary with $n>1$, then $\Psi$ is a replacement operation. Take the case $n=2$ since this is typical. Fix $V^{\prime} \in \mathbb{Q}$ then $\Phi^{\prime}(\mathbf{T})=\boldsymbol{\Psi}\left(\mathbf{V}^{\prime}, \mathbf{T}\right)$ is a unary replacement operation, whence $\mathbb{Q} \Rightarrow \Psi\left(V^{\prime}, \mathbf{T}\right)$ for all $\mathbf{T}$ such that $\mathbb{Q} \Rightarrow \mathbf{T}$ by (1). Now fix $\mathbf{V}^{\prime \prime}$ such that $\mathbb{Q} \Rightarrow \mathbf{V}^{\prime \prime}$, then $\boldsymbol{\Phi}^{\prime \prime}(\mathbf{T})=\boldsymbol{\Psi}\left(\mathbf{T}, \mathbf{V}^{\prime \prime}\right)$ is a unary replacement operation. We have seen that $\mathcal{Q} \Rightarrow \boldsymbol{\Phi}^{\prime \prime}(\mathbf{T})$ for all $\mathbf{T} \in \mathbb{Q}$. By (1) it follows that $\mathbb{Q} \Rightarrow \boldsymbol{\Phi}^{\prime \prime}(\mathbf{T})$ for all $\mathbf{T}$ such that $\mathbb{Q} \Rightarrow \mathbf{T}$. This suffices.

Another concept we need is that of types. Let $A \subset|T|$. A 1-type $\tau$ over $A$ in $T$ is a 1-point extension $U$ of $T \uparrow A$. Let $u$ be the unique vertex of $U$ not in $A$, then $v \in V \supset T \uparrow A$ is said to realize $\tau$ if the mapping, which is the identity on $A$ and takes $u$ into $v$, is an isomorphism; similarly for 2-tournaments. In the case of 2-tournaments we call $\tau i$-labeled if $u$ has label $i$.

Finally, let $T, U \in \mathcal{H}$ and $\mathscr{Q}(T), \mathcal{Q}(U)$ be strong amalgamation classes. It is easy to check that

$$
\mathcal{Q}=\left\{\mathbf{V} \in \mathscr{F}: T \text { embeds } F\left(\mathbf{V}_{0}\right) \text { and } U \text { embeds } F\left(\mathbf{V}_{1}\right)\right\}
$$

is an amalgamation class. Let $\Gamma(T, U)$ denote a homogeneous 2-tournament $\mathbf{W}$ such that $\mathbb{Q}(\mathbf{W})=\mathbb{Q}$.

We close this section with a list of notations which is adequate for $\$ \S 2-4$ and most of $\S 5$. In $\$ \S 5$ and 6 we will remind the reader where he can find an explanation of the additional notations which are required in some of the propositions.

$\begin{array}{ll}\text { notation } & \text { brief description or diagram } \\ \mathcal{Q}(T) & \text { class of finite tournaments embedded } \\ & \text { by } T\end{array}$



by $\mathbf{T}$

C

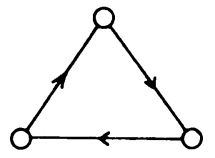

$\mathbf{C}^{i}(i<2)$

$i$-labeled copy of $C$

$C[\mathbf{X}, \mathbf{Y}, \mathbf{Z}]$

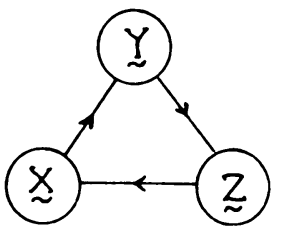

D

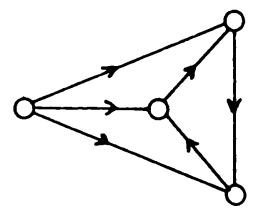

$\mathbf{D}^{i}(i<2)$

$i$-labeled copy of $D$

$D[\mathbf{X}, \mathbf{Y}, \mathbf{Z}, \mathbf{W}]$

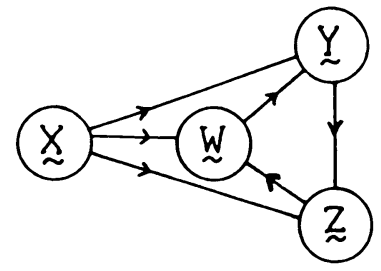

$\mathbf{E}\left(i_{0} \cdots i_{k}\right)$

transitive 2-tournament of order $k+1$ whose $j$ th vertex dominates all previous vertices and has label $i_{j}$ tournament obtained by deleting labels from the vertices of $T$

$\mathbf{F}$

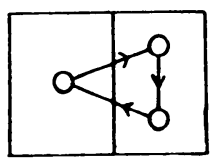
class of finite 2-tournaments 
G

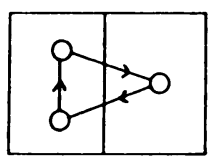

$\Gamma(T, U) \quad$ homogeneous 2-tournament embedding just those $\mathbf{X} \in \mathcal{F}$ such that $T$

$\mathcal{H}$ embeds $F\left(\mathbf{X}_{0}\right)$ and $U$ embeds $F\left(\mathbf{X}_{1}\right)$ class of countable homogeneous tournaments

$\mathscr{C}$ class of countable homogeneous 2-tournaments

$I^{i}(i<2)$ one-point tournament $i$-labeled copy of $I$

$J$

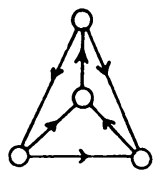

L

class of transitive tournaments

$\mathcal{L}$

$L^{n}$

class of transitive 2-tournaments

$\mathbf{L}^{n, i}(i<2)$ transitive tournament or order $n$ $P$ $i$-labeled copy of $L^{n}$ the homogeneous tournament embedding $C$ and $L^{3}$ but not $D$ $i$-labeled copy of $P$ 2-tournament obtained by labeling the vertices of $P$ so that $\left|\mathbf{P}_{i}\right|$ is dense in $P$ for $i<2$ tournament formed by the rationals with usual ordering

$\mathbf{Q}^{i}$

$\mathbf{Q}$

$R$

$\mathbf{R}$

T

T

$\bar{T}$ or $\overline{\mathbf{T}}$ $i$-labeled copy of $Q$

2-tournament obtained by labeling the vertices of $Q$ so that $\left|\mathbf{Q}_{i}\right|$ is dense in $Q$ for $i<2$ homogeneous tournament embedding every $T \in \mathscr{F}$

homogeneous 2-tournament embedding every $\mathbf{T} \in \mathcal{F}$ class of countable tournaments class of countable 2-tournaments obtained from $T$ or $\mathbf{T}$ by reversing the dominance relation 


\begin{tabular}{|c|c|}
\hline$\tilde{\mathbf{T}}$ & $\begin{array}{l}\text { obtained from } \mathbf{T} \text { by switching } \\
\text { the labels } 0 \text { and } 1\end{array}$ \\
\hline $\mathbf{T}^{i}$ & an $i$-labeled copy of $T$ \\
\hline $\mathbf{T}_{i}$ & $\begin{array}{l}\text { 2-tournament obtained from } \mathbf{T} \\
\text { by deleting all }(1-i) \text {-labeled } \\
\text { vertices }\end{array}$ \\
\hline $\mathbf{T}(i)$ & $\begin{array}{l}\text { 2-tournament obtained from } \mathbf{T} \\
\text { by adjoining a new } i \text {-labeled vertex } \\
\text { dominating all vertices in } \mathbf{T}\end{array}$ \\
\hline$T(*)$ & $\begin{array}{l}\text { tournament obtained from } T \text { by } \\
\text { adjoining a new vertex dominating all } \\
\text { vertices in } T\end{array}$ \\
\hline$T[t \rightarrow]$ or $\mathbf{T}[t \rightarrow]$ & $\begin{array}{l}\text { obtained from } T \text { or } \mathbf{T} \text { by restricting } \\
\text { to vertices dominated by } t\end{array}$ \\
\hline$T[\rightarrow t]$ or $\mathbf{T}[\rightarrow t]$ & $\begin{array}{l}\text { obtained from } T \text { or } \mathbf{T} \text { by restricting } \\
\text { to vertices dominating } t\end{array}$ \\
\hline$(\mathbf{T})$ & $\begin{array}{l}\text { tournament } U \text { obtained from } F(\mathbf{T}) \\
\text { by adjoining a new vertex } u \text { such that } \\
U[u \rightarrow]=F\left(\mathbf{T}_{0}\right) \text { and } U[\rightarrow u]=F\left(\mathbf{T}_{1}\right)\end{array}$ \\
\hline$(T, t)$ & $\begin{array}{l}\text { 2-tournament } \mathbf{U} \text { obtained from } T \text { by } \\
\text { deleting } t \text { and letting } \\
\left|\mathbf{U}_{0}\right|=|T[t \rightarrow]| \text { and }\left|\mathbf{U}_{1}\right|=|T[\rightarrow t]|\end{array}$ \\
\hline $\mathbf{T}^{\leftrightarrow}$ & $\begin{array}{l}\text { 2-tournament obtained from } \mathbf{T} \text { by } \\
\text { reversing dominance relation between } \\
\text { vertices with different labels }\end{array}$ \\
\hline$(\mathbf{T}: t)$ & $\begin{array}{l}\text { 2-tournament obtained from } \mathbf{T} \text { and } \\
t \in \mathbf{T}_{1-i} \text { by restricting to }\left|\mathbf{T}_{i}\right| \text { and } \\
\text { giving vertices in } \mathbf{T}_{i}[t \rightarrow], \mathbf{T}_{i}[\rightarrow t] \\
\text { labels } 0,1, \text { respectively }\end{array}$ \\
\hline$(\mathbf{T}: i)$ & $\begin{array}{l}\text { a copy of }(\mathbf{T}: t) \text { if the isomorphism } \\
\text { type of }(\mathbf{T}: t) \text { does not depend on } \\
\text { the choice of } t \in \mathbf{T}_{1-i}\end{array}$ \\
\hline$T[U], \mathbf{T}[U], T[\mathbf{U}]$ & wreath products \\
\hline$[T, U]$ & \\
\hline$[\mathbf{T}, \mathbf{U}]$ & \\
\hline$\Rightarrow$ & $\begin{array}{l}\text { implication between a set of } \\
\text { tournaments or 2-tournaments and } \\
\text { another such set }\end{array}$ \\
\hline
\end{tabular}


2. 2-tournaments omitting $\mathbf{D}^{0}$ and $\mathbf{D}^{1}$. We shall repeat the classification of homogeneous tournaments omitting $D$ made by Woodrow [10] and then classify homogeneous 2-tournaments which omit both $\mathbf{D}^{0}$ and $\mathbf{D}^{1}$. We shall require two facts to be proved in $\S 4$ :

(1) any homogeneous tournament embedding $L^{3}$ is infinite,

(2) $D$ and $\bar{D}$ are equivalent.

The former appears in [10] and the latter is an observation made by R. Morrow in unpublished notes.

2.1. Theorem (Woodrow). If $T \in \mathcal{H}$ omits $D$ then $T$ is isomorphic to one of $I, C$, $P, Q$.

Proof. Since any tournament of order $\geqslant 4$ embeds $L^{3}$. The only finite homogeneous tournaments are $I$ and $C$. Suppose $T \in \mathscr{H}$ is infinite and omits $D$. If $T$ omits $C$ then $T$ is transitive and by homogeneity we have $T \approx Q$. If $T$ embeds $C$, fix $t \in T$. Since $T$ omits $D$ and $\bar{D},(T, t)^{\leftrightarrow}$ is transitive. Denote $(T, t)^{\leftrightarrow}$ by $\mathbf{U}$. Since $T$ embeds $L^{3}, \mathbf{U}_{0}$ and $\mathbf{U}_{1}$ have order $>1$ whence $\left(\mathbf{U}_{0}\right) \approx\left(\mathbf{U}_{1}\right) \approx Q$. From $1.3(1), T$ embeds $J$. Interpreting this appropriately we see that each of $\mathbf{U}_{0}, \mathbf{U}_{1}$ is dense in the other. Thus $\mathbf{U} \approx \mathbf{Q}$. In $\S 1$ it was observed that $(P, p)^{\leftrightarrow} \approx \mathbf{Q}$ for any $p \in P$. Therefore $T \approx P$, which completes the proof.

2.2. Theorem. If $\mathbf{T} \in \mathcal{H}$ omits $\mathbf{D}^{0}$ and $\mathbf{D}^{1}$ then up to a variant, $\mathbf{T}$ is one of the following: $\mathbf{P}, \mathbf{Q},\left[\mathbf{T}_{0}, \mathbf{T}_{1}\right], \Gamma\left(F\left(\mathbf{T}_{0}\right), F\left(\mathbf{T}_{1}\right)\right)$.

Remark. From 2.1, for $i<2, F\left(\mathbf{T}_{i}\right)$ is isomorphic to one of $I, C, P, Q$. In fact, all possibilities are realized except that if $\mathbf{T} \approx \Gamma\left(F\left(\mathbf{T}_{0}\right), F\left(\mathbf{T}_{1}\right)\right)$ then both $\mathbf{T}_{0}$ and $\mathbf{T}_{1}$ must be infinite.

Proof. Let $\mathbf{T} \in \mathcal{K}$ omit $\mathbf{D}^{0}$ and $\mathbf{D}^{1}$. Without loss of generality suppose that $\mathbf{T}$ is not a variant of $\left[\mathbf{T}_{0}, \mathbf{T}_{1}\right]$. Then $\mathbf{T}$ embeds $\mathbf{E}(01)$ and $\mathbf{E}(10)$. Hence, $\mathbf{T}_{0}$ and $\mathbf{T}_{1}$ have cardinality $>1$. From 1.3(2), $\mathbf{T}$ embeds $\mathbf{E}(001)$ and, hence, also its variants, e.g. $\mathbf{E}(100)$. Therefore, $\left\|\mathbf{T}_{0}\right\|>3$ and similarly, $\left\|\mathbf{T}_{1}\right\|>3$. From $2.1, \mathbf{T}_{i} \in\left\{\mathbf{P}^{i}, \mathbf{Q}^{i}\right\}(i<2)$. Suppose $\mathbf{T}_{1} \approx \mathbf{Q}^{1}$. If $\mathbf{T}$ omits $\mathbf{F}$, then each vertex of $\mathbf{T}_{0}$ defines a cut in $\mathbf{T}_{1}$. In this case $\mathbf{T}$ is a variant of $\mathbf{Q}$. Thus we may suppose that $\mathbf{T}$ embeds both $\mathbf{F}$ and its variant $\mathbf{E}(101)$. It follows that, for each $t \in \mathbf{T}_{0}$, both $\left|\mathbf{T}_{1}[t \rightarrow]\right|$ and $\left|\mathbf{T}_{1}[\rightarrow t]\right|$ are dense in $\mathbf{T}_{1}$. Thus $\mathbf{T}$ embeds every $\mathbf{U} \in \mathcal{F}$ such that $\left|\mathbf{U}_{0}\right|=1$ and $\left|\mathbf{U}_{1}\right|$ is transitive. From 1.4(2) it follows that, if $\tau$ is a 0-labeled 1-type over $A \subset\left|\mathbf{T}_{1}\right|$, then $\tau$ is realized by a dense subset of $\mathbf{T}_{0}$. By 1.2 it follows that $\mathbf{T}$ embeds every $\mathbf{U} \in \mathcal{F}$ such that $\mathbf{U}_{i}$ is embeddable in $\mathbf{T}_{i}$ for $i<2$. Hence $\mathbf{T} \approx \Gamma\left(F\left(\mathbf{T}_{0}\right), F\left(\mathbf{T}_{1}\right)\right)$. This completes the case $\mathbf{T}_{1} \approx \mathbf{Q}^{1}$.

Now suppose that $\mathbf{T}_{1} \approx \mathbf{P}^{1}$. Since $\mathbf{E}(110)$ is a variant of $\mathbf{E}(001), \mathbf{T}_{1}$ embeds it. If $\mathbf{T}$ omits $\mathbf{C}^{\mathbf{l}}(0)$, then each vertex of $\mathbf{T}_{0}$ defines a cut in $\mathbf{T}_{1}$. In this case, $\mathbf{T}$ is a variant of $\mathbf{P}$. Thus, without loss of generality, suppose that $\mathbf{T}$ embeds both $\mathbf{C}^{\mathbf{l}}(0)$ and $\overline{\mathbf{C}^{\prime}(0)}$. It follows that, for each $t \in \mathbf{T}_{0}$, both $\left|\mathbf{T}_{1}[t \rightarrow]\right|$ and $\left|\mathbf{T}_{1}[\rightarrow t]\right|$ are dense in $\mathbf{T}_{1}$. We proceed as in the case $T_{1}=\mathbf{Q}^{1}$ using the obvious analogue of 1.4(2).

We close this section with a result which will be useful when we come to classify homogeneous 2-tournaments in $§ 6$. 
2.3. TheOrem. Let $\mathbf{T} \in \mathcal{H}$ embed $\mathbf{E}(01)$ and $\mathbf{E}(10)$, and $i<2$.

(1) If $\mathbf{T}_{i} \approx \mathbf{Q}^{i}$, then either $(\mathbf{T}: i) \approx \mathbf{Q}$ or $\mathbf{T}$ is a variant of $\mathbf{Q}$.

(2) If $\mathbf{T}_{i} \approx \mathbf{P}^{i}$, then either $(\mathbf{T}: i) \approx \mathbf{P}$ or $\mathbf{T}$ is a variant of $\mathbf{P}$.

Proof. (1) Without loss of generality suppose $i=0$. Let $t \in \mathbf{T}_{1}$. Since $\mathbf{T}$ embeds $\mathbf{E}(01)$ and $\mathbf{E}(10), \mathbf{T}_{0}[t \rightarrow]$ and $\mathbf{T}_{0}[\rightarrow t]$ are both nonempty. If $\mathbf{T}_{0}[t \rightarrow]$ and $\mathbf{T}_{0}[\rightarrow t]$ are both dense in $\mathbf{T}_{0}$, then $(\mathbf{T}: 1) \approx \mathbf{Q}$ and we are done. Thus suppose, without loss of generality, that $\mathbf{T}_{0}[t \rightarrow]$ is not dense in $\mathbf{T}_{0}$. There is a neighbourhood of $\mathbf{T}_{0}$ contained in $\mathbf{T}_{0}[\rightarrow t]$. By homogeneity no $a \in \mathbf{T}_{0}[t \rightarrow]$ separates two members of $\mathbf{T}_{0}[\rightarrow t]$ in the order of $\mathbf{T}_{0}$. Thus, if $a \in \mathbf{T}_{0}[t \rightarrow]$, either $a$ dominates every member of $\mathbf{T}_{0}[\rightarrow t]$, and every $b \in \mathbf{T}_{0}$ which dominates $a$ is in $\mathbf{T}_{0}[t \rightarrow]$, or every member of $\mathbf{T}_{0}[\rightarrow t]$ dominates $a$ and every $b \in \mathbf{T}_{0}$ dominated by $a$ is in $\mathbf{T}_{0}[t \rightarrow]$. Clearly, $\mathbf{T}_{0}[t \rightarrow]$ also contains a neighbourhood of $\mathbf{T}_{0}$, whence no $b \in \mathbf{T}_{0}[\rightarrow t]$ can separate two members of $\mathbf{T}_{0}[t \rightarrow]$. It follows that one of $\mathbf{T}_{0}[t \rightarrow]$ and $\mathbf{T}_{0}[\rightarrow t]$ is a cut in $\mathbf{T}_{0}$. From this we see that, with each $t \in \mathbf{T}_{1}$, we can associate a unique cut in $\mathbf{T}_{0}$. From the homogeneity of $\mathbf{T}$, distinct members of $\mathbf{T}_{1}$ yield distinct cuts in $\mathbf{T}_{0}$ and the set of all cuts associated with members of $\mathbf{T}_{1}$ is dense in $\mathbf{T}_{0}$. From the homogeneity of $\mathbf{T}$ the dominance relation on $\mathbf{T}_{1}$ induced by that on $\mathbf{T}_{0}$ must be either the same as, or the converse of, that obtained from $\mathbf{T}$; similarly, for the dominance relation between $\mathbf{T}_{0}$ and $\mathbf{T}_{1}$. Hence, up to a variant, $\mathbf{T}$ is $\mathbf{Q}$.

(2) Again suppose $i=0$ and let $t \in \mathbf{T}_{1}$. The argument is almost the same as in (1). The only point we check is that if a neighbourhood of $\mathbf{T}_{0}$ is contained in $\mathbf{T}_{0}[\rightarrow t]$, then $\mathbf{T}_{0}[\rightarrow t]$ is a cut in $\mathbf{T}_{0}$. Since in $P$ the complement of a cut is a cut, $\mathbf{T}_{0}[t \rightarrow]$ is also a cut. By homogeneity, any neighbourhood of $\mathbf{T}_{0}$ with endpoints in $\mathbf{T}_{0}[\rightarrow t]$ is contained in $\mathbf{T}_{0}[\rightarrow t]$ and so the first condition in the definition of cut is certainly satisfied. For the second condition, towards a contradiction suppose $a \in \mathbf{T}_{0}$, and that $\mathbf{T}_{0}[a \rightarrow]$ is contained in one of $\mathbf{T}_{0}[\rightarrow t]$ and $\mathbf{T}_{0}[t \rightarrow]$.

Case 1. $\mathbf{T}_{0}[a \rightarrow] \subseteq \mathbf{T}_{0}[\rightarrow t]$.

Subcase A. $a \in \mathbf{T}_{0}[\rightarrow t]$. Choose $b, c \in \mathbf{T}_{0}$ such that $a$ dominates $b, b$ dominates $c$, and $c$ dominates $a$. Since $b \in \mathbf{T}_{0}[a \rightarrow], b \in \mathbf{T}_{0}[\rightarrow t]$ and so $a, b$ realize the same type over $\{t\}$. Thus $\mathbf{T}_{0}[b \rightarrow] \subseteq \mathbf{T}_{0}[\rightarrow t]$. Similarly, $c \in \mathbf{T}_{0}[\rightarrow t]$ and $\mathbf{T}_{0}[c \rightarrow] \subseteq \mathbf{T}_{0}[\rightarrow t]$. Since $\mathbf{T}_{0}$ does not embed $\mathbf{D}^{0}$,

$$
\left|\mathbf{T}_{0}\right| \subseteq\left|\mathbf{T}_{0}[a \rightarrow]\right| \cup\left|\mathbf{T}_{0}[b \rightarrow]\right| \cup\left|\mathbf{T}_{0}[c \rightarrow]\right| .
$$

Therefore $\mathbf{T}_{0}=\mathbf{T}_{0}[\rightarrow t]$, which contradicts the embeddability of $\mathbf{E}(01)$ in $\mathbf{T}$.

Subcase B. $a \in \mathbf{T}_{0}[t \rightarrow]$. By homogeneity, for any $b \in \mathbf{T}_{0}[t \rightarrow]$ we have $\mathbf{T}_{0}[b \rightarrow] \subseteq$ $\mathbf{T}_{0}[\rightarrow t]$. Hence $b \notin \mathbf{T}_{0}[\rightarrow a]$ since $a \notin \mathbf{T}_{0}[\rightarrow t]$. Thus $\mathbf{T}_{0}[t \rightarrow]=\{a\}$. Choose $c \in$ $\mathbf{T}_{0}[\rightarrow a], d \in \mathbf{T}_{0}[a \rightarrow]$ and $\sigma \in$ aut $\mathbf{T}$ such that $\sigma(c)=d$ and $\sigma(t)=t$. Since $\sigma(t)=t$ and $\left|\mathbf{T}_{0}[t \rightarrow]\right|=\{a\}, \sigma(a)=a$. Since $c$ dominates $a, d=\sigma(c)$ dominates $a=\sigma(a)$, a contradiction. Thus Case 1 is impossible.

Case 2. $\mathbf{T}_{0}[a \rightarrow] \subseteq \mathbf{T}_{0}[t \rightarrow]$. Then $\mathbf{T}_{0}[t \rightarrow]$ contains a neighbourhood of $\mathbf{T}_{0}$ and so we can repeat the argument of Case 1 with the dominance relation between $\mathbf{T}_{0}$ and $T_{1}$ reversed.

This completes the proof that $\mathbf{T}_{0}[\rightarrow t]$ is a cut in $\mathbf{T}_{0}$ and the proof of the theorem. 
3. Proof of the main theorem. In this section we set out the proof of the principal result of the paper, omitting the proofs of three lemmas which are deferred because of their complexity. For the remainder of the paper let

$$
\mathscr{B}=\left\{\mathbf{D}^{0}, \mathbf{D}^{1}, \mathbf{E}(01), \mathbf{E}(10)\right\} \text {. }
$$

The three lemmas to be proved later are

3.1. $D \Rightarrow \mathscr{B}$,

3.2. $\mathscr{G} \Rightarrow \mathbf{T}(\mathrm{T} \in \mathcal{L})$,

3.3. $\mathscr{B} \Rightarrow C[\mathbf{S}, \mathbf{T}, \mathbf{U}](\mathbf{S}, \mathbf{T}, \mathbf{U} \in \mathscr{B})$.

Taking these results for granted we proceed as follows.

3.4. If $\mathscr{B} \Rightarrow \mathbf{S}, \mathbf{T}, \mathbf{U}, \mathbf{V}$ then $\mathscr{G} \Rightarrow D[\mathbf{S}, \mathbf{T}, \mathbf{U}, \mathbf{V}]$.

Proof. From $3.3, \mathscr{B} \Rightarrow[\mathbf{S}, \mathbf{T}]$ for all $\mathbf{S}, \mathbf{T} \in \mathscr{B}$. By $1.5(2), \mathscr{B} \Rightarrow[\mathbf{S}, \mathbf{T}]$ for all 2-tournaments $\mathbf{S}$, $\mathbf{T}$ such that $\mathscr{B} \Rightarrow \mathbf{S}$, T. Similarly, $\mathscr{B} \Rightarrow C[\mathbf{S}, \mathbf{T}, \mathbf{U}]$ for all 2-tournaments $\mathbf{S}, \mathbf{T}, \mathbf{U}$ implied by $\mathscr{B}$. The desired conclusion follows at once from the observation that

$$
D[\mathbf{S}, \mathbf{T}, \mathbf{U}, \mathbf{V}] \approx[\mathbf{S}, C[\mathbf{T}, \mathbf{U}, \mathbf{V}]] .
$$

3.5. $D \Rightarrow V$ for every $V \in \mathscr{F}$ which can be partitioned into tournaments $X, Y$ such that $X$ is transitive and $Y \approx D$.

Proof. Let $V \in \mathcal{F}$ be the disjoint union of subtournaments $X, Y$ such that $X$ is transitive and $Y \approx D$. Let $T \in \mathcal{H}$ embed $D$. We must show that $T$ embeds $V$.

Let $\|X\|=n<\omega$. We now follow the proof of 1.4(1). We recall the definition of $V\{i\}$. Let $\gamma_{0}, \ldots, \gamma_{m-1}$ be an enumeration without repetitions of all one-one mappings from $|X|$ into $r(n)$. Let $p=m+r(n)$ and $a \notin|Y| \times p$. For each $i<r(n)$ let $V\{i\}$ be the tournament defined by

$$
|V\{i\}|=\{a\} \cup(|Y| \times p), \quad V\{i\} \uparrow(|Y| \times p)=Y\left[L^{p}\right]
$$

and

$$
\begin{aligned}
& a \text { dominates }(y, j) \text { in } V\{i\} \equiv[(m \leqslant j<p \& j=m+i) \text { or } \\
& \left.\quad\left(j<m \& i \in \operatorname{rng} \gamma_{j} \& \gamma_{j}^{-1}(i) \text { dominates } y \text { in } V\right)\right] .
\end{aligned}
$$

Notice that there exist $\mathbf{X}, \mathbf{Y}, \mathbf{Z}, \mathbf{W} \in \mathcal{E}$ such that

$$
(V\{i\}, a) \approx D[\mathbf{X}, \mathbf{Y}, \mathbf{Z}, \mathbf{W}] .
$$

Thus $\mathscr{B} \Rightarrow(V\{i\}, a)$ by 3.2 and 3.4 , whence $\mathscr{B} \Rightarrow V\{i\}$ by definition of $\Rightarrow$ between a set of 2-tournaments and a set of tournaments. Using 3.1 and transitivity of $\Rightarrow$ we have $D \Rightarrow V\{i\}$ and so $T$ embeds $V\{i\}$ for each $i<r(n)$. The rest of the proof is the same as that of $1.4(1)$.

We now come to the main result.

3.6. THEOREM. (1) $D \Rightarrow T(T \in \mathscr{F})$.

(2) Up to isomorphism the only countable homogeneous tournaments are $I, C, P, Q$ and $R$. 
Proof. From 1.1 and 2.1 it is enough to prove (1).

Fix $Z \in \mathcal{H}$ which embeds $D$. Let $\mathcal{Q}$ be the class of all $T \in \mathcal{F}$ such that $Z$ embeds every $U \in \mathcal{F}$ which may be partitioned into tournaments $V, W$ with $V \approx T$ and $W \in \mathcal{L}$. From 3.5, $D \in \mathbb{Q}$, and $\mathbb{Q}$ is clearly closed under isomorphism and subtournaments. For a contradiction argument suppose $\mathscr{Q}$ is not closed under amalgamation, then we have $X, Y \in \mathbb{Q}, x \in|X|, y \in|Y|$ such that

$$
\begin{gathered}
x \neq y, \quad|X|-\{x\}=|Y|-\{y\}, \quad(X, x) \neq(Y, y), \\
X \uparrow(|X| \cap|Y|)=Y \uparrow(|X| \cap|Y|),
\end{gathered}
$$

and neither of the tournaments

$$
\begin{gathered}
R=\left\langle|X| \cup|Y|, E_{X} \cup E_{Y} \cup\{(x, y)\}\right\rangle, \\
S=\left\langle|X| \cup|Y|, E_{X} \cup E_{Y} \cup\{(y, x)\}\right\rangle
\end{gathered}
$$

is in $Q$. There exist extensions $R^{*}, S^{*}$ of $R, S$, respectively, such that

$$
R^{*} \uparrow\left(\left|R^{*}\right|-|R|\right) \text { and } S^{*} \uparrow\left(\left|S^{*}\right|-|S|\right)
$$

are finite transitive tournaments and neither $R^{*}$ nor $S^{*}$ is embeddable in $Z$. Without loss of generality suppose that

$$
\left(\left|R^{*}\right|-|R|\right) \cap\left(\left|S^{*}\right|-|S|\right)=\varnothing .
$$

Let $X^{*}$ be the tournament such that

$$
\left|X^{*}\right|=\left(\left|R^{*}\right|-\{y\}\right) \cup\left(\left|S^{*}\right|-|S|\right),
$$

every $r \in\left|R^{*}\right|-|R|$ dominates every $s \in\left|S^{*}\right|-|S|$, and all other edges are directed as specified by $R^{*}$ or $S^{*}$. Let $Y^{*}$ be similarly defined with

$$
\left|Y^{*}\right|=\left(\left|R^{*}\right|-|R|\right) \cup\left(\left|S^{*}\right|-\{x\}\right),
$$

where, again, every $r \in\left|R^{*}\right|-|R|$ dominates every $s \in\left|S^{*}\right|-|S|$. Then $X^{*}$ and $Y^{*}$ are both embeddable in $Z$ because $X$ and $Y$ are both in $\mathcal{Q}$. Amalgamating $X^{*}$ and $Y^{*}$ over their common part the amalgamated tournament embeds either $R^{*}$ or $S^{*}$, whence $Z$ embeds either $R^{*}$ or $S^{*}$. From the contradiction we conclude that $\mathcal{Q}$ is an amalgamation class. Since $D \in \mathcal{Q}$ we have $T \in \mathcal{Q}$ for every $T$ implied by $D$.

From 3.1 and 3.2, $D$ implies every tournament of order four. Let $T \in \mathcal{F}$ be of least order not implied by $D$. Let the tournament $Z$ of the last paragraph be chosen not embedding $T$. Deleting a vertex of $T$ we obtain $T^{-}$such that $D \Rightarrow T^{-}$. From above $T^{-} \in \mathcal{Q}$. But $T$ may be partitioned into $T^{-}$and a singleton which is trivially transitive. Therefore $Z$ embeds $T$. This contradiction completes the proof of the theorem.

4. A base for the transitive 2-tournaments. In this section we establish some straightforward implications for 2-tournaments concluding with the result that $\{E(0101)\}$ is a base for the class of finite transitive 2-tournaments. Simultaneously, we establish two implications for tournaments.

4.1. (R. E. WoOdRow [10]) $L^{3} \Rightarrow L^{n}(n \geqslant 1)$. 
Proof. By Ramsey's Theorem it is sufficient to show that there is no finite $T \in \mathcal{H}$ embedding $L^{3}$. For proof by contradiction let $T \in \mathcal{H}$ be finite and of least possible order embedding $L^{3}$. By homogeneity each vertex of $T$ has the same score pair $(n, n)$, where $\|T\|=2 n+1$. Clearly, $n>1$ since $C$ does not embed $L^{3}$. For each $t \in T, T[t \rightarrow]$ is homogeneous. Since every tournament of order $\geqslant 4$ embeds $L^{3}$ we have $n=\|T[t \rightarrow]\| \leqslant 3$. It follows that $n=3$ and that $T[t \rightarrow] \approx T[\rightarrow t] \approx C$. Fix $t$ and let $\alpha$ be an automorphism of $T$ such that $\alpha(T[t \rightarrow])=T[\rightarrow t]$. Then $\alpha(t)$ dominates each $u \in T[\rightarrow t]$. Clearly $\alpha(t) \in T[t \rightarrow]$ whence $\alpha(t)$ has score pair $(4,2)$. This contradiction completes the proof.

4.2. (1) $\mathbf{E}(10), \mathbf{E}(01), \mathbf{C}^{1} \Rightarrow \mathbf{E}(101), \mathbf{F}$.

(2) $\mathbf{E}(10), \mathbf{E}(01) \Rightarrow \mathbf{E}(00), \mathbf{E}(11)$.

(3) $D$ and $\bar{D}$ are equivalent.

Proof. (1) For proof by contradiction let $\mathbf{T} \in \mathcal{H}$ embed $\mathbf{E}(10), \mathbf{E}(01)$ and $\mathbf{C}^{1}$ but not $\mathbf{E}(101)$. Amalgamating $\mathbf{E}(10)$ and $\mathbf{E}(01)$ over a 0-labeled singleton (see Figure 6) we see that $\mathbf{T}$ embeds $\mathbf{F}$.

Amalgamating $\mathbf{F}$ and $\mathbf{C}^{\mathbf{l}}$ over a common edge (see Figure 7) we see that $\mathbf{T}$ embeds $\mathbf{E}(101)$ as required. Reversing the broken edge in Figure 6 and the 1-labeled 3-cycle in Figure 7 we have the conclusion that $\mathbf{E}(10), \mathbf{E}(01), \mathbf{C}^{\mathbf{l}} \Rightarrow \mathbf{F}$.

(2) Amalgamating $\mathbf{E}(10)$ and $\mathbf{E}(01)$ over a 1-labeled singleton yields $\mathbf{E}(10)$, $\mathbf{E}(01) \Rightarrow \mathbf{E}(00)$. Taking the dual we get $\mathbf{E}(11)$.

(3) From (1) we have

$$
(\mathbf{E}(10)),(\mathbf{E}(01)),\left(\mathbf{C}^{1}\right) \Rightarrow(\mathbf{E}(101))
$$

whence $\bar{D} \Rightarrow D$. Taking complements we have $D \Rightarrow \bar{D}$.

4.3. (1) $\mathbf{E}(011), \mathbf{E}(101) \Rightarrow \mathbf{E}(1011)$.

(2) $D \Rightarrow D(*)$.

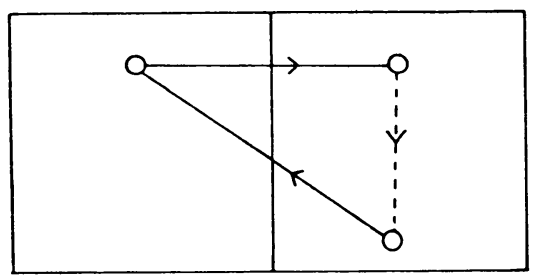

Figure 6

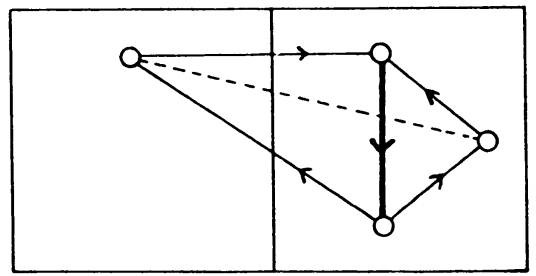

FIGURE 7 
Proof. Let $\mathbf{T} \in \mathcal{X}$ embed $\mathbf{E}(011)$ and $\mathbf{E}(101)$. For a contradiction argument suppose T does not embed $\mathbf{E}(1011)$. Amalgamating $\mathbf{E}(011)$ and $\mathbf{E}(101)$ over $\mathbf{E}(01)$ we see that $\mathbf{T}$ embeds $\mathbf{U}$ shown in Figure 8. Amalgamating $\mathbf{E}(101)$ and $\mathbf{U}$ over $\mathbf{E}(01)$ we see that $\mathbf{T}$ embeds a 2-tournament $\mathbf{V}$ shown in Figure 9 where the broken edge $c d$ may have either direction; we do not know which. Since $\mathbf{V}$ embeds $\mathbf{L}^{3,1}$ so does $\mathbf{T}$. Amalgamating $\mathbf{L}^{3,1}$ and $\mathbf{E}(101)$ over $\mathbf{L}^{2,1}$ we see that $\mathbf{T}$ embeds $\mathbf{W}$ shown in Figure 10. Notice that $\mathbf{W}$ is unchanged if we reverse the bottom edge on the right. Finally, amalgamating $\mathbf{V}$ and $\mathbf{W}$ over $\mathbf{E}(110)$ we see that $\mathbf{T}$ embeds a 2-tournament $\mathbf{X}$ depicted in Figure 11. Since $\mathbf{X}$ embeds $\mathbf{E}(1011)$ whatever the directions of the broken edges, we have the desired conclusion.

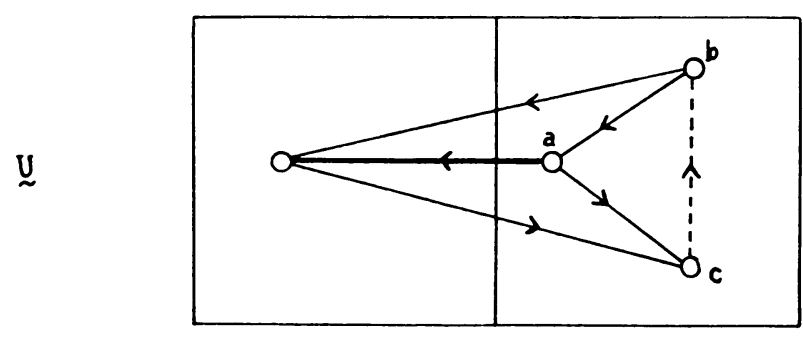

FIGURE 8

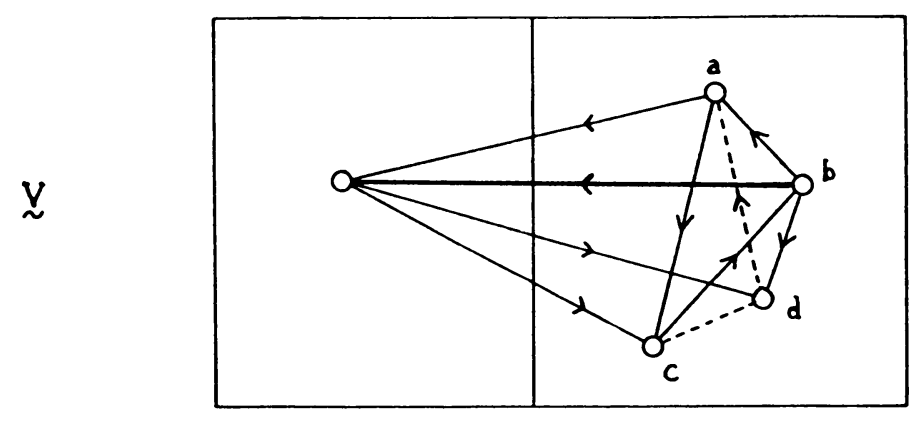

FIGURE 9

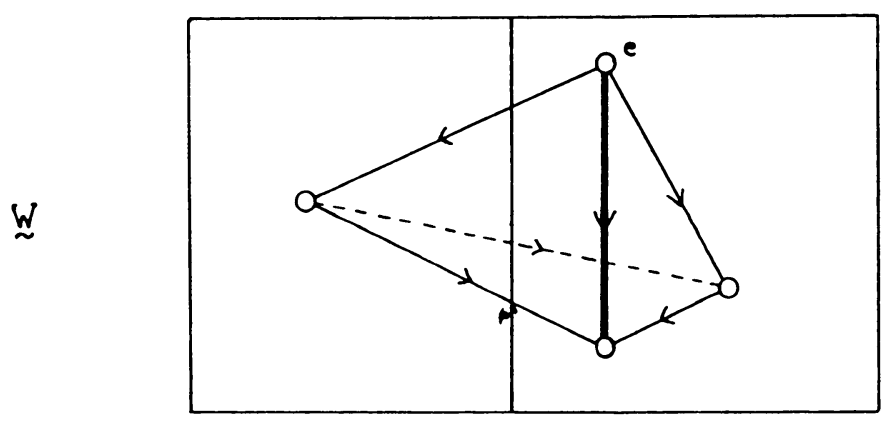

FIGURE 10 


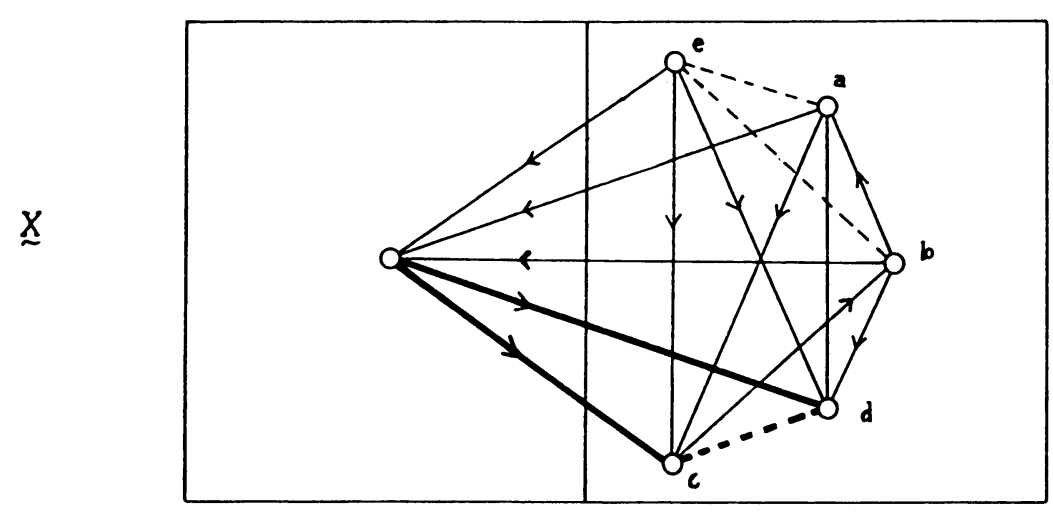

FIGURE 11

(2) From (1) we have $(\mathbf{E}(011)),(\mathbf{E}(101)) \Rightarrow(\mathbf{E}(1011))$ which is $L^{4}, D \Rightarrow D(*)$. From 4.1, $D \Rightarrow L^{4}$ whence $D \Rightarrow D(*)$.

4.4. (1) $\mathbf{E}(10), \mathbf{E}(01), \mathbf{C}^{1} \Rightarrow \mathbf{E}(010)$.

(2) $\mathbf{E}(010), \mathbf{E}(101) \Rightarrow\left(\mathbf{E}(1010)\right.$ or $\left.\mathbf{C}^{1}\right)$, i.e., every $\mathbf{T} \in \mathcal{H}$ embedding both $\mathbf{E}(010)$ and $\mathbf{E}(101)$ embeds either $\mathbf{E}(010)$ or $\mathbf{C}^{1}$.

Proof. (1) Consider $\mathbf{T} \in \mathcal{K}$ embedding $\mathbf{E}(10), \mathbf{E}(01)$ and $\mathbf{C}^{\mathbf{l}}$. If $\mathbf{T}$ embeds $\mathbf{C}^{0}$ then $\mathbf{T}$ embeds $\mathbf{E}(010)$ from $4.2(1)$. Thus, suppose $\mathbf{T}$ does not embed $\mathbf{C}^{0}$. It follows that $\mathbf{T}_{0} \approx \mathbf{Q}^{0}$. If $\mathbf{T}$ does not embed $\mathbf{E}(010)$, then each $t \in \mathbf{T}_{1}$ determines a cut in $\mathbf{T}_{0}$ because every vertex in $\mathbf{T}_{0}[t \rightarrow]$ dominates every vertex in $\mathbf{T}_{0}[\rightarrow t]$. By homogeneity the left section of such a cut has no greatest element, and the right section has no least element. It follows that $\mathbf{T}_{1} \approx \mathbf{Q}^{1}$, contradicting $\mathbf{C}^{1}$ being embeddable in $\mathbf{T}$. Therefore, $\mathbf{T}$ embeds $\mathbf{E}(010)$ as required.

(2) Let $\mathbf{T} \in \mathcal{X}$ embed $\mathbf{E}(010)$ and $\mathbf{E}(101)$ but neither $\mathbf{C}^{\prime}$ nor $\mathbf{E}(1010)$. Then $\mathbf{T}_{1} \approx \mathbf{Q}^{1}$. Amalgamating $\mathbf{E}(010)$ and $\mathbf{E}(101)$ over $\mathbf{E}(01)$ as in Figure 12 we see that $\mathbf{T}$ embeds $\mathbf{F}$. Since $\mathbf{T}$ also embeds $\mathbf{E}(101)$ it follows easily from the transitivity of $\mathbf{T}_{1}$ that $\mathbf{T}$ embeds both the tournaments shown in Figure 13. Amalgamating these tournaments over $\mathbf{L}^{3,0}$ we see that $\mathbf{T}$ embeds $\mathbf{E}(1010)$. This contradiction completes the proof.

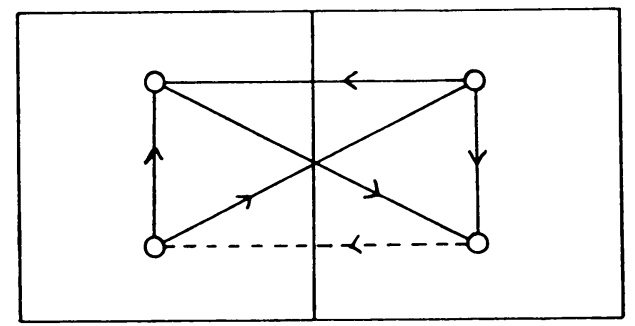

Figure 12 

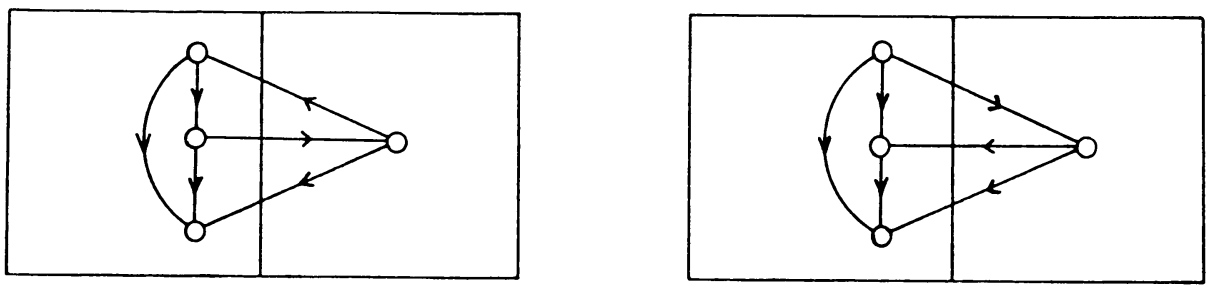

FIGURE 13

4.5. $\mathbf{E}(0101) \Rightarrow \mathbf{E}(10101)$.

Proof. Applying 1.5(1) to 4.4(1) with $\boldsymbol{\Phi}(\mathbf{T})=\left[\mathbf{T}, \mathbf{I}^{1}\right]$ (1) we obtain

$$
\mathbf{E}(1101), \mathbf{E}(1011), \overline{\mathbf{D}}^{1}(1) \Rightarrow \mathbf{E}(10101) \text {. }
$$

Applying 1.5(1) to 4.4(2) with $\boldsymbol{\Phi}(\mathbf{T})=\mathbf{T}(1)$,

$$
\mathbf{E}(0101), \mathbf{E}(1011) \Rightarrow\left(\mathbf{E}(10101) \text { or } \mathbf{D}^{1}\right) \text {. }
$$

Applying 1.5(1) to 4.2(2) with $\boldsymbol{\Phi}(\mathbf{T})=\mathbf{T}(0)(1)$,

$$
\mathbf{E}(1001), \mathbf{E}(0101) \Rightarrow \mathbf{E}(1101) \text {. }
$$

Also, since $\mathbf{E}(100)$ is a variant of $\mathbf{E}(001)$, from 1.3(2) we get $\mathbf{E}(01), \mathbf{E}(10) \Rightarrow \mathbf{E}(100)$. Applying 1.5(1) again,

$$
\mathbf{E}(011), \mathbf{E}(101) \Rightarrow \mathbf{E}(1001) \text {. }
$$

Combining this with $(*)$ we have $\mathbf{E}(0101) \Rightarrow \mathbf{E}(1101)$. From 4.3(1) $\mathbf{E}(0101) \Rightarrow \mathbf{E}(1011)$. From $4.2(3), \mathbf{D}^{1} \Rightarrow \overline{\mathbf{D}}^{1}$, whence $\mathbf{D}^{1}(1) \Rightarrow \overline{\mathbf{D}}^{1}(1)$ by $1.5(1)$ with $\Phi(\mathbf{T})=\mathbf{T}(1)$. From 4.3(2), $\mathbf{D}^{1} \Rightarrow \mathbf{D}^{1}(1)$, whence $\mathbf{D}^{1} \Rightarrow \overline{\mathbf{D}}^{1}(1)$. The first two implications of the proof now yield

$$
\mathbf{E}(0101), \mathbf{D}^{1} \Rightarrow \mathbf{E}(10101) \text { and } \mathbf{E}(0101) \Rightarrow\left(\mathbf{E}(10101) \text { or } \mathbf{D}^{1}\right) .
$$

The desired conclusion follows immediately.

$$
\text { 4.6. } \mathbf{E}(0101) \Rightarrow \mathbf{E}(\sigma)\left(\sigma \in \in^{<\omega} 2\right) \text {. }
$$

Proof. We proceed by induction on the length of $\sigma$. Suppose $\mathbf{E}(0101) \Rightarrow \mathbf{E}(\tau)$ for some $\tau \in^{<\omega} 2$. Applying 1.5(1), $\mathbf{E}(01010) \Rightarrow \mathbf{E}\left(\tau^{\cap} 0\right)$. From 4.5 we have

$$
\mathbf{E}(0101) \Rightarrow \mathbf{E}(1010) \Rightarrow \mathbf{E}(01010) \text {. }
$$

Thus $\mathbf{E}(0101) \Rightarrow \mathbf{E}\left(\tau^{\cap} 0\right)$ and similarly, $\mathbf{E}(0101) \Rightarrow \mathbf{E}\left(\tau^{\cap} 1\right)$. This completes the proof.

5. Verification of the main lemmas. For 3.1 we must show that $D \Rightarrow \Re$. Since $(\mathbf{E}(01)) \approx L^{3}$ and $(\mathbf{E}(10)) \approx C, D \Rightarrow \mathbf{E}(01), \mathbf{E}(10)$ trivially. From $4.3(2), D \Rightarrow D(*) \approx$ $\left(\mathbf{D}^{0}\right)$ and so $D \Rightarrow \mathbf{D}^{0}$. From $4.2(3), D \Rightarrow \bar{D}$. By the analogue of $1.5(1)$ for tournaments $D(*) \Rightarrow \bar{D}(*)$, whence $D \rightarrow \bar{D}(*) \approx\left(\mathbf{D}^{1}\right)$ by transitivity. This completes the proof.

For 3.2 we must show that $\mathscr{B} \Rightarrow \mathbf{T}(\mathbf{T} \in \mathcal{L})$. In $1.3(2)$ reverse the arrows between vertices having different labels and then take the dual to get

$$
\mathbf{E}(01), \mathbf{E}(11) \Rightarrow \mathbf{E}(011) \text {. }
$$

Combining this with $4.2(2)$ we see that $\mathscr{B} \Rightarrow \mathbf{E}(011)$. Also from $4.2(1)$ we have $\mathscr{B} \Rightarrow \mathbf{E}(101)$. From 4.4(1) and 1.5(1) with $\boldsymbol{\Phi}(\mathbf{T})=\mathbf{T}(1)$,

$$
\mathbf{E}(101), \mathbf{E}(011), \mathbf{D}^{1} \Rightarrow \mathbf{E}(0101) \text {. }
$$


Combining these implications $\mathscr{B} \Rightarrow \mathbf{E}(0101)$ and so, from 4.6 , $\mathscr{B}$ implies every transitive 2-tournament.

The verification of 3.3 will occupy the remainder of this section and requires thirteen auxiliary propositions. One obvious, but nonetheless useful, observation is that if $\mathscr{B} \Rightarrow \mathbf{T}$ then $\mathscr{B} \Rightarrow \mathbf{U}$ for every variant $\mathbf{U}$ of $\mathbf{T}$.

\subsection{If $T \in \mathscr{F}$ may be partitioned into two transitive tournaments then, $D \Rightarrow T$.}

Proof. From 3.1 and $3.2, D \Rightarrow(T)(T \in \mathcal{L})$. From this it is clear that 5.1 is true when one side of the partition is a singleton. By 1.4(1) the general conclusion follows.

5.2. If $\mathbf{T} \in \mathcal{H}$ embeds $\mathbf{E}(01)$ and $\mathbf{E}(10)$, then either $\mathbf{T}$ is a variant of either $\mathbf{P}$ or $\mathbf{Q}$, or $\mathbf{T}$ embeds every $\mathbf{L} \in \mathcal{L}$.

Proof. Let $\mathbf{T}$ satisfy the hypothesis. By homogeneity, $\mathbf{T}_{0}$ and $\mathbf{T}_{1}$ are both infinite, because if $\mathbf{T}_{i}$ is finite then a nontrivial equivalence relation can be defined on $\mathbf{T}_{i-1}$, which is impossible. If $\mathbf{T}$ embeds both $\mathbf{D}^{0}$ and $\mathbf{D}^{1}$ the conclusion is immediate from 3.2. Otherwise, by 2.1 there exists $i<2$ such that $\mathbf{T}_{i} \approx \mathbf{P}^{i}$ or $\mathbf{T}_{i} \approx \mathbf{Q}^{i}$. Suppose that $\mathbf{T}$ is neither a variant of $\mathbf{P}$ nor a variant of $\mathbf{Q}$. From $2.3,(\mathbf{T}: i)$ is isomorphic to either $\mathbf{P}$ or $\mathbf{Q}$. Therefore, $\mathbf{T}$ embeds every 2-tournament which can be partitioned into an $(i-1)$-labeled singleton and an $i$-labeled transitive tournament. By 1.4(2), switching labels if necessary, $\mathbf{T}$ embeds every $\mathbf{L} \in \mathfrak{L}$.

$$
\text { 5.3. If } \mathbf{U} \in \mathcal{F}, i<2,\left\|\mathbf{U}_{i}\right\| \leqslant 2 \text {, and } \mathbf{U}_{1-i} \in \mathcal{L} \text {, then } \mathscr{G} \Rightarrow \mathbf{U} \text {. }
$$

Proof. Since $\mathscr{B}$ is invariant under switching labels, we can suppose $i=0$. From 1.4(2) we can suppose $\left\|\mathbf{U}_{0}\right\|=1$. Let $\mathbf{T} \in \mathcal{K}$ embed $\mathscr{B}$. We have to show that $(\mathbf{T}: 1)$ embeds $\mathcal{L}$. Since $\mathbf{T}$ embeds $\mathbf{D}^{1}$, by $5.1, F\left(\mathbf{T}_{1}\right)$ embeds every tournament which can be partitioned into two transitive tournaments. Hence $(T: 1)$ is neither a variant of $\mathbf{P}$ nor of $\mathbf{Q}$. By 4.2(1), $\mathbf{T}$ embeds $\mathbf{E}(101)$ and $\mathbf{F}$, whence $(\mathbf{T}: 1)$ embeds $\mathbf{E}(01)$ and $\mathbf{E}(10)$. By $5.2,(\mathbf{T}: 1)$ embeds $\mathcal{L}$ as required.

\subsection{If $\mathscr{B} \Rightarrow \mathbf{T}$, then $\mathscr{B} \Rightarrow \mathbf{T}(i)(i<2)$.}

Proof. From 1.5(2) it suffices to show that $\mathscr{B} \Rightarrow \mathbf{T}(i)(i<2)$ for each $\mathbf{T} \in \mathscr{B}$. From $4.3(2), \mathscr{B} \Rightarrow \mathbf{D}^{i}(i)$, and from $3.2, \mathscr{B} \Rightarrow \mathbf{E}(01 i), \mathbf{E}(10 i)$. It remains to show that $\mathscr{B} \Rightarrow \mathbf{D}^{1-i}(i)$. Let $\mathbf{T} \in \mathcal{H}$ embed $\mathscr{B}$ and, for proof by contradiction, suppose $\mathbf{T}$ does not embed $D^{1}(0)$. Consider the 2-tournament $U$ shown in Figure 14 where the direction of the edge $a b$ is not yet specified. By 3.2, $\mathbf{T}$ embeds each transitive 2-tournament. Hence, $\mathbf{T}$ embeds $\mathbf{U}\{\{a, d, e, f\}$. Since $\mathbf{T}$ also embeds $\mathbf{U} \uparrow$ $\{c, d, e, f\} \approx \mathbf{D}^{1}, \mathbf{T}$ embeds $\mathrm{U} \uparrow\{a, c, d, e, f\}$ by amalgamation. If the edge $c a$ were directed the other way we should have $\mathbf{D}^{\mathbf{l}}(0)$. Since $\mathbf{U}\{\{b, c, d, e, f\} \approx \mathbf{U}$ । $\{a, c, d, e, f\}$, it is also embeddable in T. Amalgamating $\mathbf{U}\{\{b, c, d, e, f\}$ and $\mathbf{U}\{\{a, c, d, e, f\}$ we see that $\mathbf{U}$ is embeddable in $\mathbf{T}$ provided the edge $a b$ is suitably directed. Let $\mathbf{V}$ be obtained from $\mathbf{U}$ by reversing the direction of the edges $c d$, de, and replacing $d$ by $g$. Since $V_{1}$ is transitive $V$ is embeddable in $T$ by 5.3. Amalgamating $\mathbf{U}$ and $\mathbf{V}$ we see that either $c d g$ or $d e g$ is a copy of $\mathbf{C}^{\mathbf{l}}$ and so $\mathbf{T}$ embeds $\mathbf{D}^{\mathbf{l}}(0)$ by restricting to $\{b, c, d, f, g\}$ or $\{a, d, e, f, g\}$, respectively. 


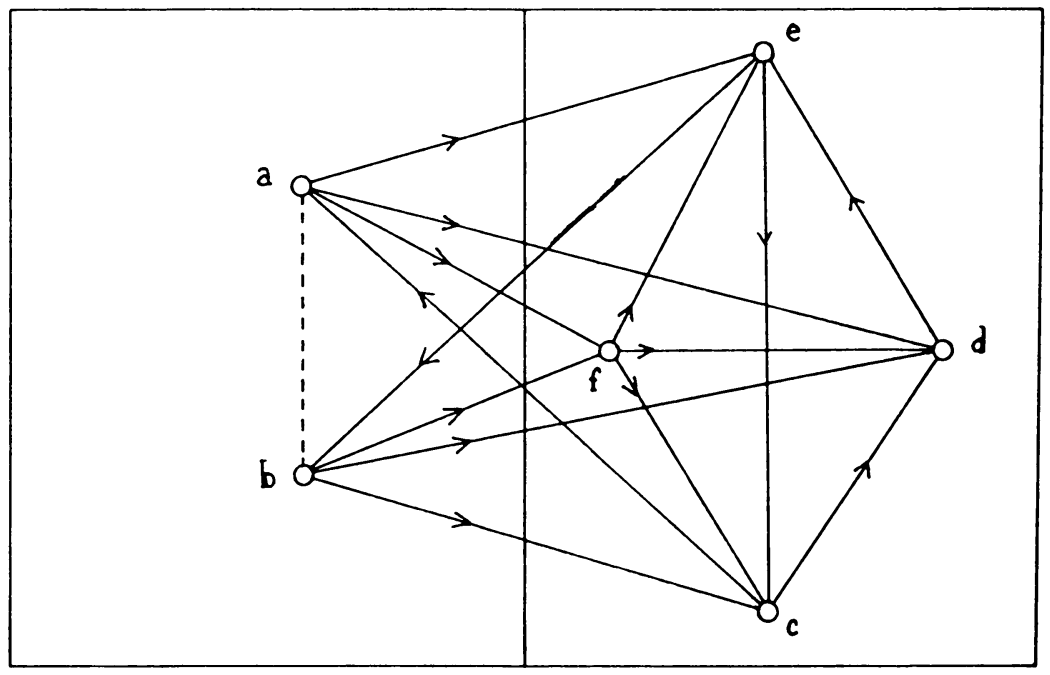

FIGURE 14

Using 5.4 and the same method of proof as for 5.4 we obtain

5.5. If $i<2, \mathbf{U} \in \mathscr{F},\left|\mathbf{U}_{i}\right|=\{a\}$ and $\mathscr{B} \Rightarrow(\mathbf{U}: a)$, then $\mathscr{B} \Rightarrow \mathbf{U}$.

Our next aim is to show that $\mathscr{B} \Rightarrow\left[\mathbf{C}^{i}, \mathbf{C}^{1-i}\right]$. At this point the reader should review the definitions of $\left\langle\left\langle\mathbf{C}^{i}, \mathbf{T}\right\rangle\right\rangle$ and $\left\langle\left\langle\mathbf{C}^{i}, \mathbf{U}\right\rangle\right\rangle^{-1}$ found in $\S 1$. As a preliminary, define

$$
\mathcal{C}=\left\{\mathbf{W} \in \mathscr{F}: \mathscr{B} \Rightarrow\left\langle\left\langle\mathbf{C}^{i}, \mathbf{W}\right\rangle\right\rangle, i<2\right\}
$$

5.6. $\mathcal{L} \subset \mathcal{C}$.

Proof. Let $\mathbf{T} \in \mathcal{K}$ embed $\mathscr{B}$, and $\mathbf{U}$ denote $\left\langle\left\langle\mathbf{C}^{i}, \mathbf{T}\right\rangle\right\rangle^{-1} \in \mathcal{H}$. We must show that $\mathrm{U}$ embeds every transitive 2-tournament.

Claim 1. $F(\mathbf{U})$ embeds every $W \in \mathscr{F}$ which can be partitioned into two transitive subtournaments.

To see this, let $W \in \mathscr{F}$ be partitionable into two transitive subtournaments and $\mathbf{W}^{1-i}$ be a $(1-i)$-labeled copy of $W$. By $5.1 \mathbf{T}$ embeds $\mathbf{W}^{1-i}$ since it embeds $\mathbf{D}^{1-i}$. By 5.4, $\mathbf{T}$ embeds $\mathbf{W}^{1-i}(i)(i)$. Amalgamating $\mathbf{W}^{1-i}(i)(i)$ and $\mathbf{C}^{i}$ over a common edge we see that $\mathbf{U}$ embeds some $\mathbf{X}$ such that $F(\mathbf{X})=W$.

Claim 2. U embeds $\mathbf{E}(01)$ and $\mathbf{E}(10)$.

To see this suppose without loss of generality that $i=0$, and let $\mathbf{V}$ be as in Figure 15. By 5.5, $\mathbf{X}=\mathbf{V} \uparrow(|\mathbf{V}|-\{a\})$ is implied by $\mathscr{G}$ since $(\mathbf{U}: b) \approx \mathbf{F}$. Since $\mathbf{V} \approx$ $\overline{\bar{X}}(1), \mathscr{B} \Rightarrow \mathbf{V}$. Hence $\mathbf{T}$ embeds $\mathbf{V}$, and $\mathbf{U}$ embeds $\mathbf{E}(01)$. Reversing the edge $b a$ of $\mathbf{V}$ we have a variant, whence $\mathbf{U}$ also embeds $\mathbf{E}(01)$.

From Claim 1, $\mathbf{U}$ is neither a variant of $\mathbf{P}$ nor of $\mathbf{Q}$. From Claim 2 and 5.2, $\mathbf{U}$ embeds every $\mathbf{W} \in \mathcal{L}$.

5.7. $\mathscr{B} \Rightarrow\left[\mathbf{C}^{i}, \mathbf{C}^{i-1}\right](i<2)$. 


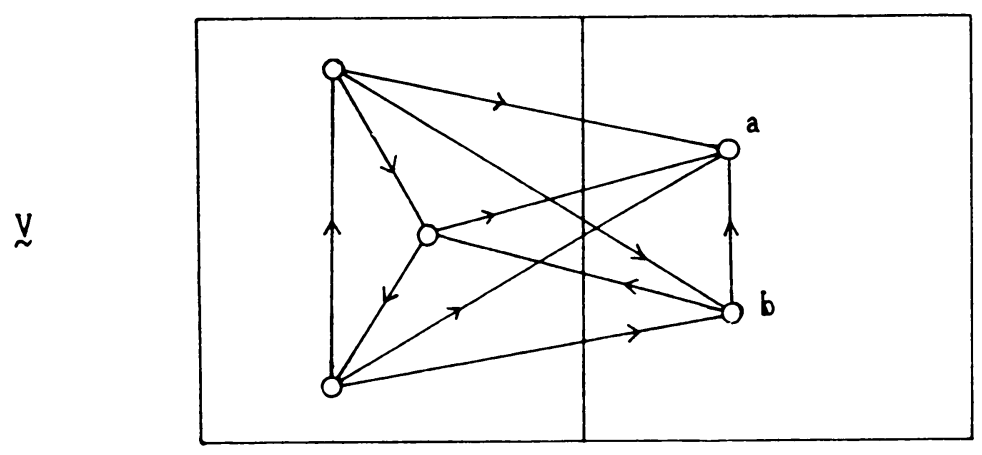

FIGURE 15

Proof. We follow the same line as in 5.4. Suppose $i=0$. Let $\mathbf{T} \in \mathcal{H C}$ embed $\mathscr{G B}$ and, for proof by contradiction, suppose $\mathbf{T}$ does not embed $\left[\mathbf{C}^{0}, \mathbf{C}^{1}\right]$. Observe that $\mathbf{T}$ embeds the tournament $\mathbf{U}$ presented in Figure 16 where the broken edge is directed one way or the other, we do not know which. To see this we argue as follows. Let the restrictions of $\mathbf{U}$ to $|\mathbf{U}|-\{a, b\}$ and $|\mathbf{U}|-\{a, c\}$ be denoted by $\mathbf{X}$ and $\mathbf{Y}$. Since $\mathbf{X} \approx \mathbf{C}^{\prime}(0)(0)$ and $\mathbf{Y} \approx \mathbf{C}^{0}(1)(1), \mathbf{X}$ and $\mathbf{Y}$ are embedded in $\mathbf{T}$ by 5.4. Now $\mathbf{Z}=$ $\mathbf{U} \mid(|\mathbf{U}|-\{a\})$ is obtained by amalgamation of $\mathbf{X}$ and $\mathbf{Y}$ over their common part. If the edge $b c$ were directed the other way we should have $\left[\mathbf{C}^{0}, \mathbf{C}^{1}\right]$. Also $\mathbf{W}=$ $\mathbf{U}$ ) $(|\mathbf{U}|-\{b\})$ being isomorphic to $\mathbf{Z}$ is also embeddable in $\mathbf{T}$. By amalgamation of $\mathbf{Z}$ and $\mathbf{W}$, if the edge $a b$ is suitably directed, then $\mathbf{T}$ embeds $\mathbf{U}$. $\mathbf{T}$ also embeds the tournament $\mathbf{S}$ shown in Figure 17 where again the broken edge is directed one way or the other; we do not know which.

The tournaments obtained from $\mathbf{S}$ by deleting $a$ and $b$, respectively, are embedded in $\mathbf{T}$ by 5.6. Whichever way the broken edge is directed in $\mathbf{S}$, the tournament $\mathbf{V}$ depicted in Figure 18 is embedded in $\mathbf{S}$ and hence in $\mathbf{T}$ where the edge $a b$ is directed the same way as in $\mathbf{U}$. Amalgamating $\mathbf{U}$ and $\mathbf{V}$ over the common subtournament with universe $\{a, b, \ldots, f\}$ we see that $\mathbf{T}$ embeds $\left[\mathbf{C}^{0}, \mathbf{C}^{1}\right]$ as required.

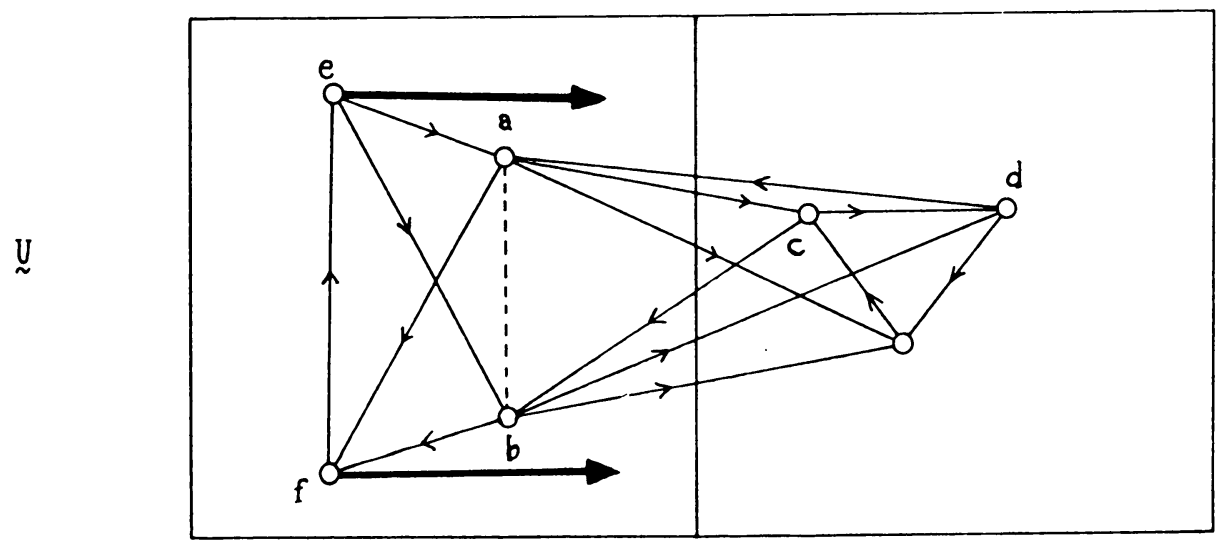

FigURE 16 


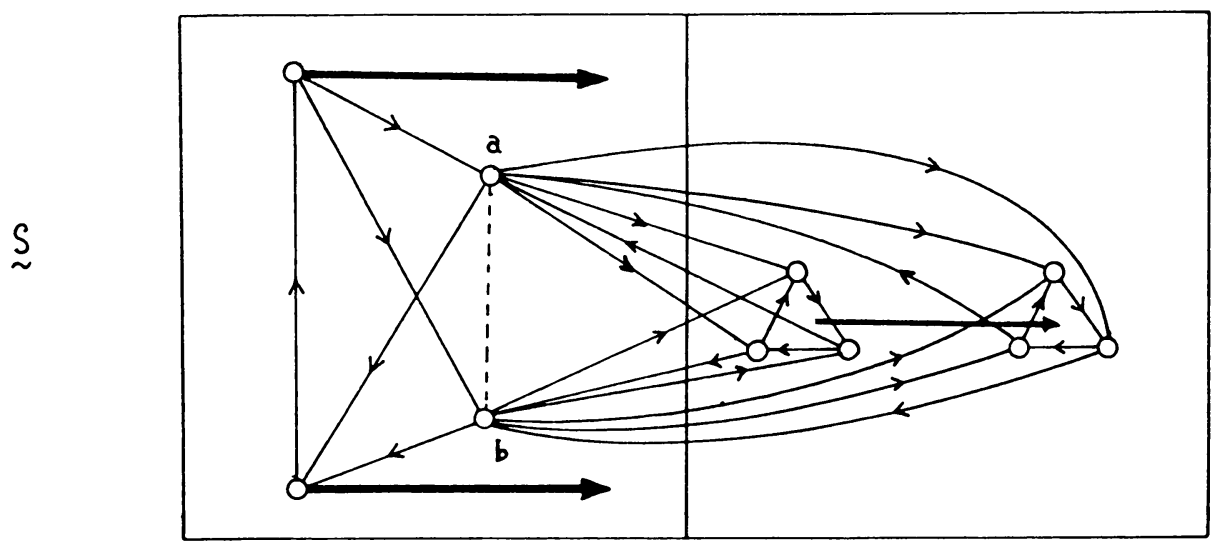

FIGURE 17

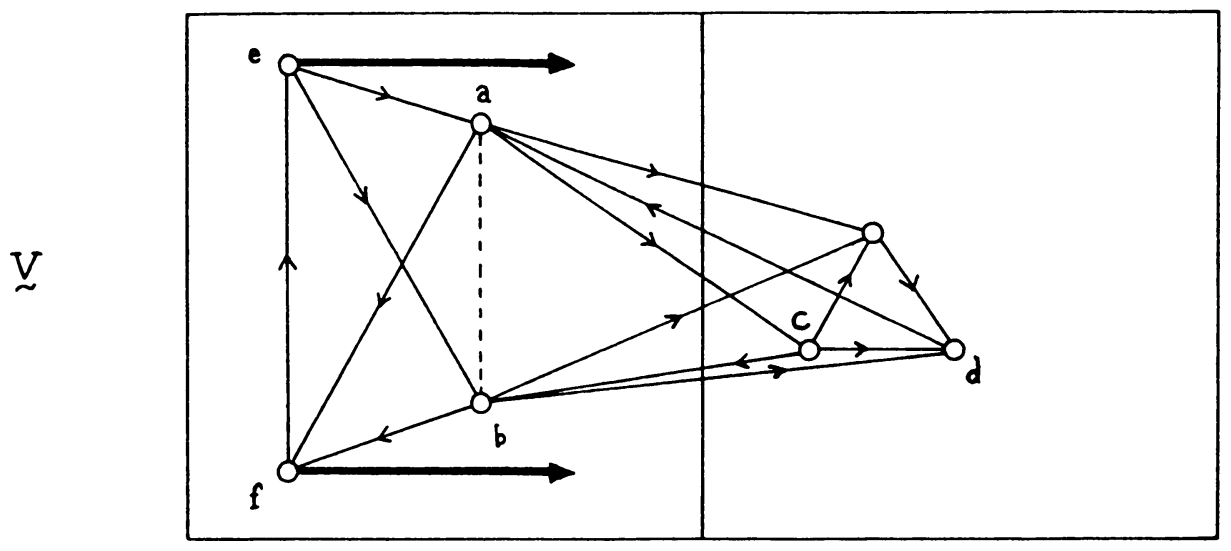

Figure 18

\subsection{If $\mathscr{B} \Rightarrow \mathbf{U}, \mathbf{V}$ then $\mathscr{B} \Rightarrow[\mathbf{U}, \mathbf{V}]$.}

Proof. By $1.5(2)$ it is sufficient to show that $\mathscr{B} \Rightarrow[\mathbf{U}, \mathbf{V}]$ for all $\mathbf{U}, \mathbf{V} \in \mathscr{B}$. From $5.1, D \Rightarrow[D, D]$, whence $\mathscr{B} \Rightarrow\left[\mathbf{D}^{i}, \mathbf{D}^{i}\right](i<2)$. From $5.7, \mathscr{B} \Rightarrow\left[\mathbf{C}^{0}, \mathbf{C}^{1}\right]$. Hence from $5.4, \mathscr{B} \Rightarrow\left[\mathbf{D}^{0}, \mathbf{C}^{1}\right] \approx\left[\mathbf{C}^{0}, \mathbf{C}^{1}\right](0)$. Since $\left[\mathbf{C}^{0}, \mathbf{D}^{1}\right]$ is a variant of $\left[\mathbf{D}^{0}, \mathbf{C}^{1}\right], \mathscr{B} \Rightarrow\left[\mathbf{C}^{0}, \mathbf{D}^{1}\right]$. By $5.4, \mathscr{B} \Rightarrow\left[\mathbf{D}^{0}, \mathbf{D}^{1}\right] \approx\left[\mathbf{C}^{0}, \mathbf{D}^{1}\right](0)$. Similarly, $\mathscr{B} \Rightarrow\left[\mathbf{D}^{1}, \mathbf{D}^{0}\right]$. From $3.2, \mathscr{B} \Rightarrow[\mathbf{U}, \mathbf{V}]$ for $\mathbf{U}, \mathbf{V} \in\{\mathbf{E}(01), \mathbf{E}(10)\}$. Each tournament of the form $[\mathbf{U}, \mathbf{V}]$, where one of $\mathbf{U}, \mathbf{V}$ is in $\{\mathbf{E}(01), \mathbf{E}(10)\}$ and the other in $\left\{\mathbf{D}^{0}, \mathbf{D}^{1}\right\}$, either has the form $\mathbf{D}^{i}(j)(k)$ or is the complement of $\overline{\mathbf{D}}^{i}(j)(k)$, where $i \in\{0,1\}=\{j, k\}$. By 5.4 each such tournament is implied by 9 . This completes the proof.

We can now strengthen 5.5 to

5.9. If $\mathbf{T} \in \mathscr{F}, i<2,\left|\mathbf{T}_{i}\right|=\{a, b\}$ and $\mathscr{B} \Rightarrow(\mathbf{T}: a)$, $(\mathbf{T}: b)$, then $\mathscr{B} \Rightarrow \mathbf{T}$.

Proof. Let $\mathbf{U}$ be a copy of $\mathbf{T}$ disjoint from $\mathbf{T}$ except that, in $\mathbf{U}, b$ plays the role of $a$ in $\mathbf{T}$ and $a$ plays the role of $b$. Obtain $\mathbf{V}$ from $\mathbf{U}$ by reversing the edge $a b$. Let $\mathbf{W}$ be the unique 2-tournament such that $|\mathbf{W}|=|\mathbf{T}| \cup|\mathbf{V}|, \mathbf{W}|| \mathbf{T}|=\mathbf{T}, \mathbf{W}||\mathbf{V}|=\mathbf{V}$ and all other edges are directed from $|\mathbf{T}|$ to $|\mathbf{V}|$. Let $\mathbf{S} \in \mathcal{H}$ embed $\mathscr{B}$, and $\mathbf{X}, \mathbf{Y}$ be obtained 
from $\mathbf{W}$ by deleting $b, a$, respectively. Since $(\mathbf{X}: a) \approx[(\mathbf{T}: a),(\mathbf{V}: a)]$ and $(\mathbf{V}: a) \approx$ $(\mathbf{T}: b)$ we have $\mathscr{B} \Rightarrow \mathbf{X}$ by 5.5 and 5.8. Similarly, $\mathscr{B} \Rightarrow \mathbf{Y}$. Thus $\mathbf{S}$ embeds $\mathbf{X}$ and $\mathbf{Y}$. By amalgamation, $\mathbf{S}$ embeds either $\mathbf{W}$ or the tournament obtained from $\mathbf{W}$ by reversing the edge $a b$. Hence, $\mathbf{S}$ embeds either $\mathbf{T}$ or $\mathbf{U}$, which completes the proof.

Before reading the rest of the section the reader is advised to study the definitions of $\langle\langle\mathbf{E}(01), \mathbf{T}\rangle\rangle,\langle\langle\mathbf{E}(10), \mathbf{T}\rangle\rangle,\left\langle\left\langle\mathbf{D}^{i}, \mathbf{T}\right\rangle\right\rangle$ and $\left(\mathbf{C}^{i}, \mathbf{T}\right)(i<2)$ to be found in $\S 1$.

5.10. If $\mathscr{B} \Rightarrow \mathbf{U}$, then $\mathscr{B} \Rightarrow\left\langle\left\langle\mathbf{D}^{i}, \mathbf{U}\right\rangle\right\rangle(i<2)$.

Proof. By $1.5(2)$ it is enough to show that $\mathscr{B} \Rightarrow\left\langle\left\langle\mathbf{D}^{i}, \mathbf{U}\right\rangle\right\rangle$ for each $\mathbf{U} \in \mathscr{B}$. Now $\mathscr{B} \Rightarrow\left\langle\left\langle\mathbf{D}^{i}, \mathbf{D}^{j}\right\rangle\right\rangle(i, j<2)$ by 5.8 , and $\mathscr{B} \Rightarrow\left\langle\left\langle\mathbf{D}^{i}, \mathbf{E}(01)\right\rangle\right\rangle,\left\langle\left\langle\mathbf{D}^{i}, \mathbf{E}(10)\right\rangle\right\rangle$ by 5.9 as required.

In the same kind of way we easily obtain

5.11. If $\mathscr{B} \Rightarrow \mathbf{U}$ then $\mathscr{B} \Rightarrow\langle\langle\mathbf{E}(01), \mathbf{U}\rangle\rangle,\langle\langle\mathbf{E}(10), \mathbf{U}\rangle\rangle$.

Another result along the same lines is

5.12. If $\mathscr{B} \Rightarrow \mathbf{U}$ then $\mathscr{B} \Rightarrow\left(\mathbf{C}^{i}, \mathbf{U}\right)(i<2)$.

$\underset{\sim}{U}$

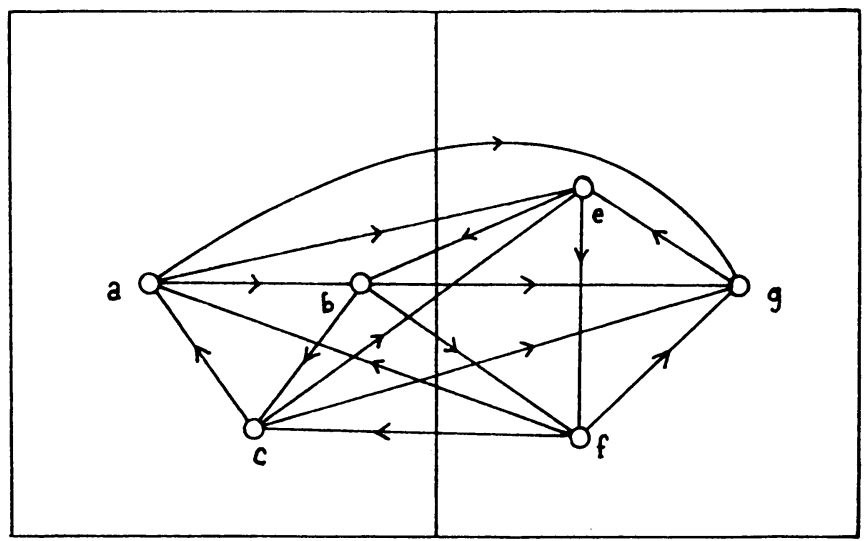

FIGURE 19

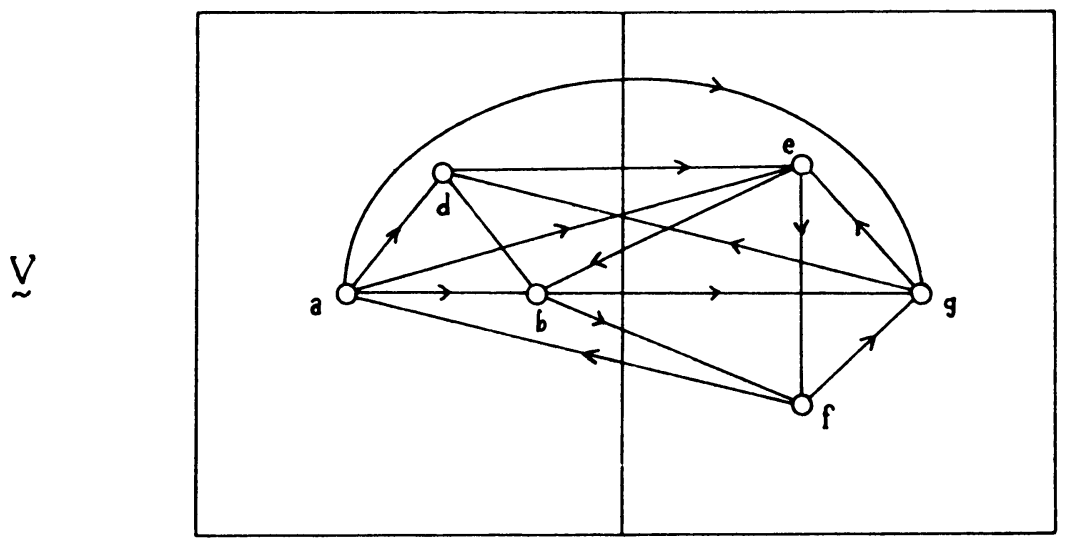

FIGURE 20 
Proof. Following the same line as in 5.10 it is enough to show that $\mathscr{B} \Rightarrow\left(\mathbf{C}^{i}, \mathbf{U}\right)$ for all $\mathbf{U} \in\left\{\mathbf{E}(01), \mathbf{E}(10), \mathbf{D}^{0}, \mathbf{D}^{1}\right\}$. We have $\mathscr{B} \Rightarrow\left(\mathbf{C}^{i}, \mathbf{D}^{j}\right)(j<2)$ by application of 5.10, and $\mathscr{B} \Rightarrow\left(\mathbf{C}^{i}, \mathbf{E}(01)\right),\left(\mathbf{C}^{i}, \mathbf{E}(10)\right)$ from 5.9.

The last result we need before checking 3.3 is

5.13. $\mathscr{B} \Rightarrow C[\mathbf{U}, \mathbf{V}, \mathbf{W}]$ for any $\mathbf{U}, \mathbf{V}, \mathbf{W} \in\{\mathbf{E}(01), \mathbf{E}(10)\}$.

Proof. From 5.13, $\mathscr{B} \Rightarrow \mathbf{U}$ the 2-tournament shown in Figure 19, because $\mathbf{U}$ can be written $\left(\mathbf{C}^{\mathrm{l}}, \mathbf{F}\right)$.
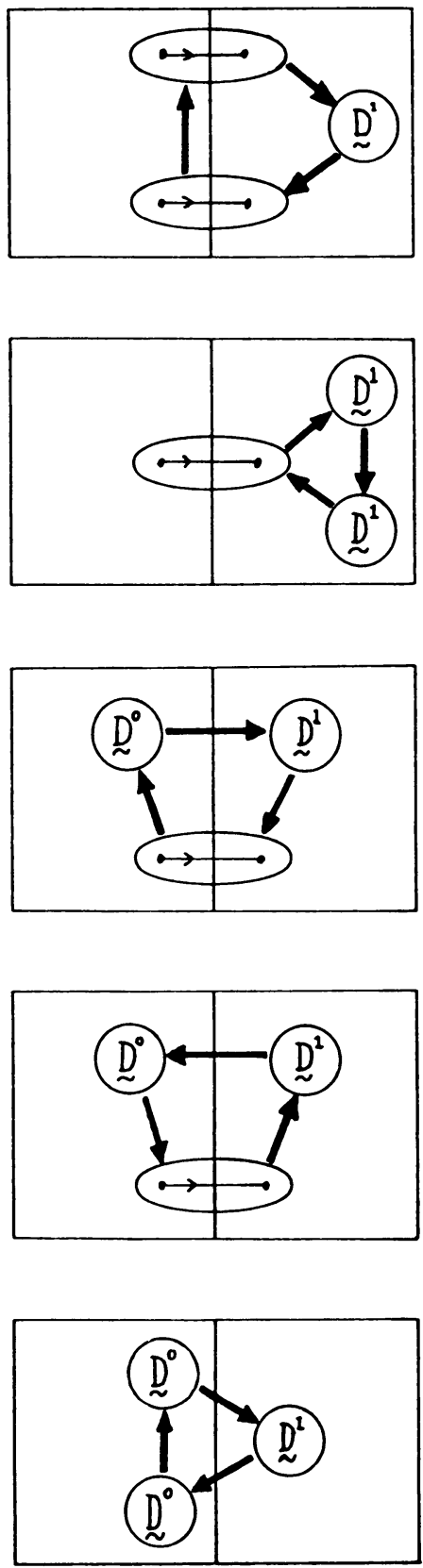

FigURE 21 

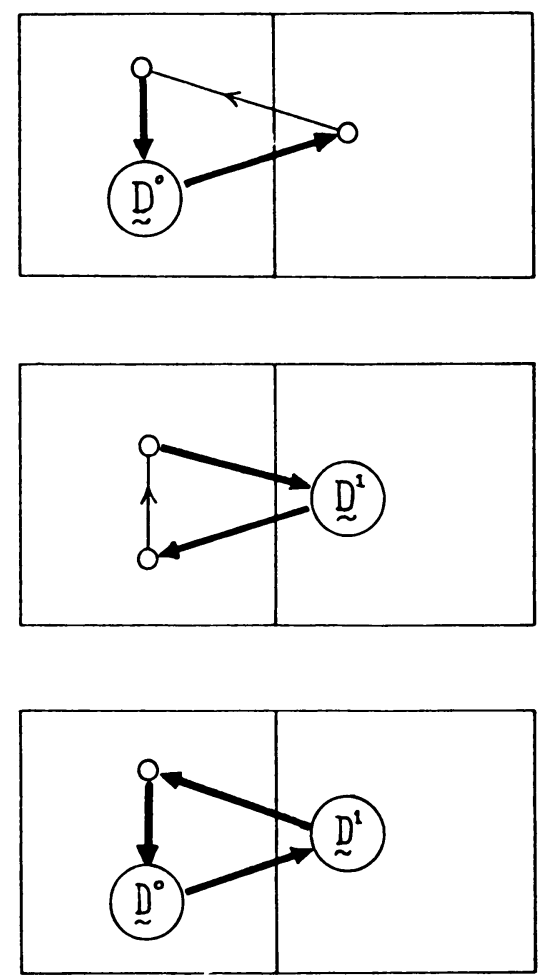

FigURE 22

Let $\mathbf{T} \in \mathcal{H}$ embed $\mathscr{B}$ and for proof by contradiction suppose $\mathbf{T}$ does not embed $C[\mathbf{E}(10)]$. Then $\mathbf{T}$ embeds $\mathbf{V}$ the 2-tournament shown in Figure 20. We see this by amalgamation with respect to the vertices $a, b$. Notice that if the edge $a b$ were reversed then $\mathbf{V}$ would become $C[\mathbf{E}(10)]$. Also, the 2-tournaments obtained from $\mathbf{V}$ by deleting $a, b$ are easily seen to be implied by $\mathscr{B}$ by application of 5.9 . Amalgamating $\mathbf{U}$ and $\mathbf{V}$ over their common part we see that $\mathbf{T}$ embeds $C[\mathbf{E}(10)]$, whence $\mathscr{B} \Rightarrow C[\mathbf{E}(10)]$. To obtain $C[\mathbf{E}(10)]$ from the amalgamated tournament, delete $a$ or $b$ according as the edge $c d$ is directed upwards or downwards. By reversing the edge de we see that $\mathscr{B} \Rightarrow C[\mathbf{E}(01), \mathbf{E}(10), \mathbf{E}(10)]$ and these two cases are clearly sufficient.

We can now prove Lemma 3.3. From the last proposition it is enough to show that $\mathscr{B} \Rightarrow C[\mathbf{U}, \mathbf{V}, \mathbf{W}]$ whenever $\mathbf{U} \in\left\{\mathbf{D}^{0}, \mathbf{D}^{1}\right\}$ and $\mathbf{V}, \mathbf{W} \in\left\{\mathbf{D}^{0}, \mathbf{D}^{1}, \mathbf{E}(01), \mathbf{E}(10)\right\}$. Rather than treat every single case, we consider five cases which are representative. We shall show that $\mathscr{B}$ implies each of the five 2-tournaments sketched in Figure 21. The first one, $C\left[\mathbf{D}^{0}, \mathbf{E}(10), \mathbf{E}(10)\right]$, is reduced to the first two 2-tournaments, pictured in Figure 22, by application of 5.9. In turn, those 2-tournaments are reduced to ones implied by $\mathscr{B}$ by 5.5 and 5.9 , respectively. All the cases in which both $\mathbf{V}, \mathbf{W} \in$ $\{\mathbf{E}(01), \mathbf{E}(10)\}$ can be treated similarly. The second 2-tournament in Figure 21, $C\left[\mathbf{D}^{1}, \mathbf{D}^{1}, \mathbf{E}(10)\right]$, is reduced to the third one in Figure 22 by application of 5.5 , and that 2-tournament is seen to be implied by $\mathscr{B}$ by application of 5.10 . This argument is good whenever just one of $\mathbf{V}$ and $\mathbf{W}$ is in $\{\mathbf{E}(01), \mathbf{E}(10)\}$. The third 2-tournament 
in Figure 21 is reduced to $\left[\mathbf{D}^{0}, \mathbf{D}^{1}\right]$ by 5.11 , and $\mathscr{B} \Rightarrow\left[\mathbf{D}^{0}, \mathbf{D}^{1}\right]$ from 5.8 . The fourth 2-tournament in Figure 21 is $C\left[\mathbf{D}^{1}, \mathbf{D}^{0}, \mathbf{E}(10)\right]$. Reversing all edges in the left box we see that the lower left vertex may be deleted by 5.4 . The remainder is implied by $\mathscr{B}$ from 5.10. The last 2-tournament in Figure 21, $C\left[\mathbf{D}^{1}, \mathbf{D}^{0}, \mathbf{D}^{0}\right]$, is reduced to $\left[\mathbf{D}^{0}, \mathbf{D}^{1}\right]$ by 5.10. This completes the verification of Lemma 3.3.

6. Homogeneous 2-tournaments. We shall show that the machinery developed above is sufficient to show that $\mathscr{B}$ is a base for $\mathscr{F}$. This enables us to complete the classification of homogeneous 2-tournaments begun in 2.2.

As a preliminary, consider the binary operation $\Xi$ on $\mathscr{F}$ defined as follows. For convenience suppose $\mathbf{U}, \mathbf{V} \in \mathscr{F}$ are disjoint and that $a$ is a new vertex. Define $\Xi(\mathbf{U}, \mathbf{V})=\mathbf{T}$ where $\left|\mathbf{T}_{0}\right|=|\mathbf{U}| \cup\{a\},\left|\mathbf{T}_{1}\right|=|\mathbf{V}|$, and $E_{\mathbf{T}}$ is

$$
E_{\mathbf{U}} \cup E_{\mathbf{V}} \cup(|\mathbf{U}| \times|\mathbf{V}|) \cup\left(\{a\} \times\left(\left|\mathbf{U}_{0}\right| \cup\left|\mathbf{V}_{0}\right|\right)\right) \cup\left(\left(\left|\mathbf{U}_{1}\right| \cup\left|\mathbf{V}_{1}\right|\right) \times\{a\}\right) .
$$

From the propositions of $\S 5$ it is not hard to verify that $\mathscr{B} \Rightarrow \Xi(U, V)$ for all $\mathbf{U}$, $\mathbf{V} \in \mathbf{B}$. We consider three particular cases as illustrations, leaving the rest to the reader. Firstly, $\mathbf{\Xi}\left(\mathbf{E}(01), \mathbf{D}^{0}\right) \approx\left[\mathbf{L}^{3,0}, \mathbf{D}^{1}\right]$, which is implied by $\mathscr{B}$ from 5.8. Secondly, $\Xi\left(\mathbf{E}(01), \mathbf{D}^{1}\right)$, shown in Figure 23, has the form $C\left[\mathbf{I}^{0}, \mathbf{I}^{0}, \mathbf{D}^{1}\right](0)$ and so is implied by $\Re$ from 3.3 and 5.4. Finally, $\Xi(\mathbf{E}(01), \mathbf{E}(10))$, shown in Figure 24 , has the form $\mathbf{X}(0)$ where $\mathbf{X}$ is a variant of $\mathbf{G}(1)$. Hence, $\mathscr{B} \Rightarrow \Xi(\mathbf{E}(01), \mathbf{E}(10))$ by $4.2(1)$ and 5.4 .

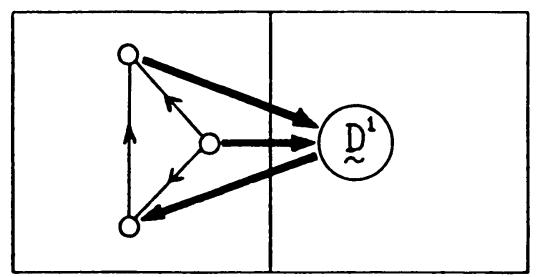

FIGURE 23

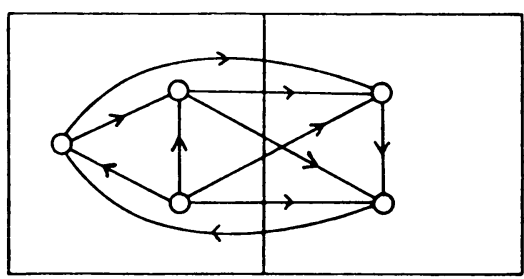

FIGURE 24

Since the principle enunciated in 1.5 applies also to the operation $\Xi$ we have

$$
\text { 6.1. If } \mathscr{B} \Rightarrow \mathbf{U}, \mathbf{V} \text { then } \mathscr{B} \Rightarrow \Xi(\mathbf{U}, \mathbf{V}) \text {. }
$$

Our aim is to show that $\mathscr{B}$ is a base for $\mathscr{F}$. To this end let $\mathbf{T} \in \mathcal{H}$ embed $\mathscr{B}$. Let $\mathscr{Q}$ denote the class

$$
\{\mathbf{U} \in \mathscr{F}:(\forall \mathbf{W} \in \mathscr{F})(\forall n<\omega)
$$

$$
\begin{aligned}
& \text { [if there exists a partition }\left\{\mathbf{V}, \mathbf{V}^{\prime}\right\} \text { of } \mathbf{W} \\
& \left.\left.\qquad \text { such that } \mathbf{V} \approx \mathbf{I}^{0} \text { and } \mathbf{V}^{\prime} \approx \mathbf{U}\left[L^{n}\right] \text {, then } \mathbf{T} \text { embeds } \mathbf{W}\right]\right\} .
\end{aligned}
$$

By an argument similar to that made in $3.6, \mathfrak{Q}$ is an amalgamation class. Further, in 3.6 it was shown that $D \Rightarrow T$ for all $T \in \mathscr{F}$. Since $\mathbf{T}$ embeds $\mathbf{D}^{0}$, $\mathbf{T}$ embeds every 0 -labeled 2-tournament, whence $\mathbf{D}^{0} \in \mathbb{Q}$. To see that $\mathbf{D}^{1} \in \mathbb{Q}$, it is enough to show that $\mathscr{B} \Rightarrow \mathbf{W}$ for every $\mathbf{W}$ which can be partitioned into $\mathbf{V}$ and $\mathbf{V}^{\prime}$ such that $\mathbf{V} \approx \mathbf{I}^{0}$ and $\mathbf{V}^{\prime} \approx \mathbf{D}^{\prime}\left[L^{n}\right]$. From 5.5 it suffices to prove that $\mathscr{B} \Rightarrow D[\mathbf{W}, \mathbf{X}, \mathbf{Y}, \mathbf{Z}]$ for all $\mathbf{W}, \mathbf{X}, \mathbf{Y}, \mathbf{Z} \in \mathcal{L}$. This fact is immediate from 3.2 and 3.4. Therefore $\mathbf{D}^{1} \in \mathbb{Q}$. Taking 
$\mathbf{U}, \mathbf{V}$ to be arbitrary transitive 2-tournaments in 6.1 we see that $\mathbf{E}(10) \in \mathbb{Q}$. By symmetry, $\mathbf{E}(01) \in \mathbb{Q}$ also. We have shown that $\mathscr{B} \subset \mathbb{Q}$.

For proof by contradiction let $\mathbf{U} \in \mathcal{F}$ be a 2-tournament of least order not implied by $\mathscr{B}$. Choose $u \in \mathbf{U}_{0}$ and let $\mathbf{U}^{-}$be obtained from $\mathbf{U}$ by deleting $u$. Then $\mathscr{B} \Rightarrow \mathbf{U}^{-}$ and so $\mathbf{U}^{-} \in \mathbb{Q}$ since $\mathbb{Q}$ is an amalgamation class. Since $\mathbf{U}$ can be partitioned into $\mathbf{U}^{-}$ and a copy of $\mathbf{I}^{0}, \mathbf{T}$ embeds $\mathbf{U}$. But $\mathbf{T}$ is an arbitrary homgeneous 2-tournament embedding $\mathscr{G}$. Therefore, $\mathscr{B} \Rightarrow \mathbf{U}$ which establishes

\section{2. $\mathscr{T}$ is a base for $\mathscr{F}$.}

A final technical result is required.

\subsection{If $\mathbf{U} \in \mathcal{F}$ and $\left\|\mathbf{U}_{0}\right\|=1$, then $\mathbf{E}(01), \mathbf{E}(10), \mathbf{D}^{1} \Rightarrow \mathbf{U}$.}

Proof. Let $\mathbf{T} \in \mathcal{K}$ embed $\mathbf{E}(01), \mathbf{E}(10)$ and $\mathbf{D}^{1}$. We have to show that $(\mathbf{T}: 1)$ embeds every $\mathbf{U} \in \mathcal{F}$. It is clear that $(\mathbf{T}: 1) \in \mathcal{H}$. Thus, by 6.2 it is enough to show that $(\mathbf{T}: \mathbf{1})$ embeds $\mathscr{B}$. The following seven claims establish this:

(i) $\mathbf{T}$ embeds $\mathbf{F}$ and $\mathbf{F}^{\rightarrow}$.

(ii) $(\mathbf{T}: 1)$ embeds $\mathbf{E}(01)$ and $\mathbf{E}(10)$.

(iii) $(\mathbf{T}: 1)$ embeds every $\mathbf{U} \in \mathcal{L}$.

(iv) $\mathbf{T}$ embeds every $\mathbf{U} \in \mathscr{F}$ such that $\mathbf{U}_{0}, \mathbf{U}_{1} \in \mathcal{L}$.

(v) ( $\mathbf{T}: 1)$ embeds $\mathbf{C}^{0}$ and $\mathbf{C}^{\prime}$.

(vi) If $\mathbf{T}$ embeds $\mathbf{U} \in \mathcal{F}$, then $\mathbf{T}$ embeds $\mathbf{U}(1)$.

(vii) (T: 1$)$ embeds $\mathbf{D}^{0}$ and $\mathbf{D}^{1}$.

Proofs of (i) - (vii). (i) By 5.2, $\mathbf{T}$ embeds $\mathbf{F}^{\leftrightarrow}$ since $\mathbf{F}^{\leftrightarrow} \in \mathcal{L}$. Since $\left\{\mathbf{E}(01), \mathbf{E}(10), \mathbf{D}^{\mid}\right\}$is closed under $\mathbf{X} \mapsto \mathbf{X}^{\leftrightarrow}, \mathbf{T}$ also embeds $\mathbf{F} \approx \mathbf{F}^{\leftrightarrow} \leftrightarrow$.

(ii) is immediate from (i).

(iii) From 5.1, $\mathrm{T}$ embeds every 1-labeled tournament which may be partitioned into two transitive tournaments. Therefore, $(\mathbf{T}: 1)$ is a variant of neither $\mathbf{P}$ nor $\mathbf{Q}$. By (ii) and 5.2, (T: 1) embeds every $\mathbf{U} \in \mathcal{L}$.

(iv) follows from (iii) and 1.4(2).

(v) Since $\left\{\mathbf{E}(01), \mathbf{E}(10), \mathbf{D}^{1}\right\}$ is closed under $\mathbf{X} \mapsto \mathbf{X}^{\leftrightarrow}$, it is enough to show that ( $\mathbf{T}: 1)$ embeds $\mathbf{C}^{0}$ or, equivalently, that $\mathbf{T}$ embeds $\mathbf{C}^{1}(0)$.

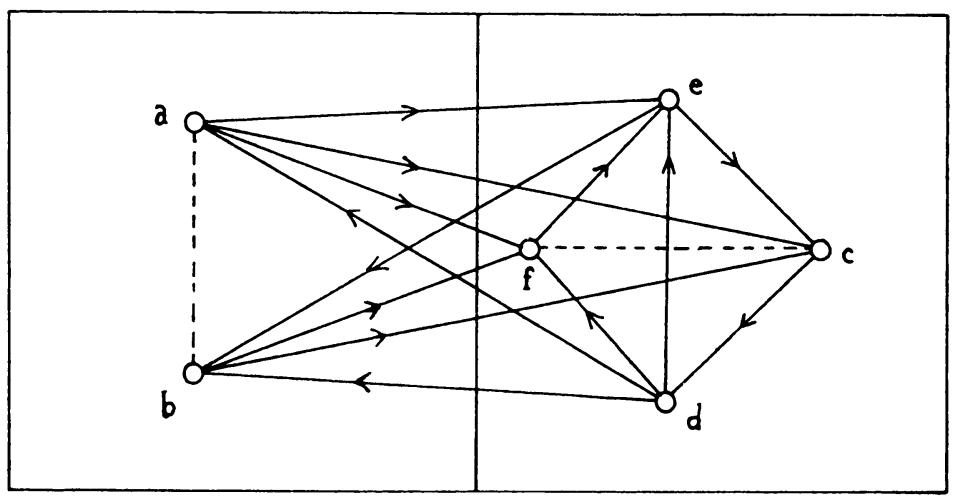

FIGURE 25 
This may be seen by examining the 2-tournament $\mathbf{U}$, shown in Figure 25, whose explanation is as follows. Firstly, $\mathbf{U}\{\{a, c, e\}$ is embeddable in $\mathbf{T}$ by (iv), and $\mathbf{U} \uparrow\{c, d, e\}$ is embeddable since $\mathbf{D}^{1}$ is. Amalgamating $\mathbf{U} \uparrow\{a, c, e\}$ and $\mathbf{U} \uparrow\{c, d, e\}$ we see that $\mathbf{U} \uparrow\{a, c, d, e\}$ is embeddable. For if the edge $d a$ were reversed we should be done. Now $\mathrm{U} \uparrow\{b, c, d, e\}$, being isomorphic to $\mathrm{U} \uparrow\{a, c, d, e\}$, is also embeddable. By amalgamation $\mathbf{T}$ embeds $\mathbf{U} \uparrow\{a, b, c, d, e\}$ provided the edge $a b$ is suitably directed. Regardless of the direction of $a b$, the tournament abdef is embedded in $\mathbf{T}$ by (iv). Amalgamating $\mathbf{U} \uparrow\{a, b, c, d, e\}$ and $\mathbf{U}\{\{a, b, d$, ef $\}$ we see that $\mathbf{T}$ embeds $\mathbf{U}$ provided the edge $c f$ is suitably directed. Whichever way $c f$ is directed, $\mathbf{U}$ embeds $\mathrm{C}^{\mathrm{l}}(0)$, and so we are done.

(vi) Since $\mathbf{U} \mapsto \mathbf{U}(1)$ is a replacement operation, by $1.5(2)$ it is enough to show that $\mathbf{T}$ embeds $\mathbf{U}(1)$ for all $\mathbf{U} \in\left\{\mathbf{E}(01), \mathbf{E}(10), \mathbf{D}^{1}\right\}$. This is apparent from (iv) and 4.3(2).

(vii) Since $\left\{\mathbf{E}(01), \mathbf{E}(10), \mathbf{D}^{1}\right\}$ is closed under the operation $\mathbf{X} \rightarrow \mathbf{X}^{\leftrightarrow}$, it is enough to show that $(\mathbf{T}: 1)$ embeds $\mathbf{D}^{1}$ or, equivalently, that $\mathbf{T}$ embeds $\mathbf{C}^{1}(0)^{\leftrightarrow}(1)$. From (vi) it suffices to show that $\mathbf{C}^{\mathbf{l}}(0)^{\hookrightarrow}$ is embedded in $\mathbf{T}$ which is clear from (v).

From (ii) and (vii), ( $\mathbf{T}: \mathbf{1})$ embeds $\mathscr{B}$, so we are done.

We can now establish the following classification of homogeneous 2-tournaments. Let $\mathbf{E}$ denote the empty 2-tournament.

6.4. TheOREM. If $\mathbf{T} \in \mathcal{H}$, then up to a variant, $\mathbf{T}$ is one of the following:

$$
\mathbf{P}, \mathbf{Q},\left[\mathbf{T}_{0}, \mathbf{T}_{1}\right], \Gamma\left(F\left(\mathbf{T}_{0}\right), F\left(\mathbf{T}_{1}\right)\right) \text {. }
$$

The possibilities for $\mathbf{T}_{i}$ are $\mathbf{E}, \mathbf{I}^{i}, \mathbf{C}^{i}, \mathbf{P}^{i}, \mathbf{Q}^{i}$ and $\mathbf{R}^{i}(i<2)$. The only constraints are that one of $\mathbf{T}_{0}$ and $\mathbf{T}_{1}$ be nonempty and that both be infinite if $\mathbf{T} \approx \Gamma\left(F\left(\mathbf{T}_{0}\right), F\left(\mathbf{T}_{1}\right)\right)$.

Proof. If $\mathbf{T}$ embeds neither $\mathbf{D}^{0}$ nor $\mathbf{D}^{1}$ we have the result by 2.2 . If either of $\mathbf{E}(01)$ or $\mathbf{E}(10)$ is not embedded in $\mathbf{T}$ we have the result by 3.6. If $\mathbf{T}$ embeds $\mathscr{G}$ we have the result by 6.2 . This leaves the case in which $\mathbf{T}$ embeds both $\mathbf{E}(01)$ and $\mathbf{E}(10)$ and one of $\mathbf{D}^{0}$ and $\mathbf{D}^{1}$ but not the other. Without loss of generality, assume $\mathbf{T}$ embeds $\mathbf{E}(01)$, $\mathbf{E}(10)$ and $\mathbf{D}^{1}$ but not $\mathbf{D}^{0}$.

Claim 1. $\mathbf{T}_{0} \approx \mathbf{P}^{0}$ or $\mathbf{T}_{0} \approx \mathbf{Q}^{0}$.

Proof. Since $\mathbf{T}_{1}$ embeds $\mathbf{D}^{1}, \mathbf{T}_{1}$ is certainly infinite. Were $\mathbf{T}_{0}$ finite, there would be distinct $u, v \in \mathbf{T}_{1}$ such that $(\mathbf{T}: u)=(\mathbf{T}: v)$. By homogeneity $(\mathbf{T}: u)=(\mathbf{T}: v)$ for all $u, v \in \mathbf{T}_{1}$. Since $\mathbf{T}$ embeds both $\mathbf{E}(01)$ and $\mathbf{E}(10)$, this contradicts homogeneity. Therefore, $\mathbf{T}_{0}$ is infinite and the claim follows by 2.1 .

Claim 2. If $a \in\left|\mathbf{T}_{0}\right|$ and $B$ is a finite subset of $\left|\mathbf{T}_{1}\right|$, there exists a dense subset $A \subset\left|\mathbf{T}_{0}\right|$ such that every $a^{\prime} \in A$ realizes the same l-type over $B$ as $a$ does.

Proof. We proceed by induction on $|B|$. If $|B|=0$ the result is obvious. Suppose the claim holds for $B$ and that $b \in\left|\mathbf{T}_{1}\right|-B$. We will prove the claim for $B \cup\{b\}$. Let $\mathbf{U}$ be the subtournament of $\mathbf{T}$ such that

$$
\left|\mathbf{U}_{0}\right|=\left\{t \in\left|\mathbf{T}_{0}\right|: \operatorname{tp}(t \mid B)=\operatorname{tp}(a \mid B) \text { in } \mathbf{T}\right\}
$$

and

$$
\left|\mathbf{U}_{1}\right|=\left\{t \in\left|\mathbf{T}_{1}\right|: \operatorname{tp}(t \mid B)=\operatorname{tp}(b \mid B) \text { in } \mathbf{T}\right\} .
$$

Since $\mathbf{T} \in \mathcal{H}$ so is $\mathbf{U}$. Since the claim holds for $B,\left|\mathbf{U}_{0}\right|$ is a dense subset of $\left|\mathbf{T}_{0}\right|$ and so $\mathbf{U}_{0} \approx \mathbf{T}_{0}$. By $6.3, \mathbf{U}$ embeds both $\mathbf{E}(01)$ and $\mathbf{E}(10)$. Since $\mathbf{T}$ embeds $\mathbf{D}^{1}, \mathbf{T}_{1} \approx \mathbf{R}^{1}$ 
and, hence, $\mathbf{U}_{1} \approx \mathbf{R}^{1}$ also. By $2.3,(\mathbf{U}: 0) \approx \mathbf{Q}$ or $(\mathbf{U}: 0) \approx \mathbf{P}$ according as $\mathbf{T}_{0}$ is $\mathbf{Q}^{0}$ or $\mathbf{P}^{0}$. This means that both $\mathbf{U}_{0}[b \rightarrow]$ and $\mathbf{U}_{1}[\rightarrow b]$ are dense in $\mathbf{U}_{0}$ and hence in $\mathbf{T}_{0}$. This confirms the claim for $B \cup\{b\}$ in place of $B$.

This completes the proof of the claim.

Returning to the proof of the theorem, from 6.3, given finite $B \subset\left|\mathbf{T}_{1}\right|$ every possible 0 -labeled 1-type over $B$ is realized in T. Further, by 6.2 each such 1-type is realized by a dense subset of $\left|\mathbf{T}_{0}\right|$. It follows by 1.2 that $\mathbf{T}$ embeds every finite $\mathbf{V}$ such that $\mathbf{V}_{0}$ is embeddable in $\mathbf{T}_{0}$. Thus $\mathbf{T}$ is $\Gamma\left[F\left(\mathbf{T}_{0}\right), F\left(\mathbf{T}_{1}\right)\right]$, which completes the proof of the theorem.

It seems extremely likely that the results obtained in this paper can be extended to $n$-tournaments for any fixed $n, 2<n<\omega$. In particular, we conjecture that, if $\mathscr{B}$ contains an $i$-labeled copy of $D$ for each $i<n$ and a copy of every tournament having exactly one vertex with each label, then $\mathscr{G}$ is a basis for the class of finite $n$-tournaments.

\section{REFERENCES}

1. R. Fraissé, Sur l'extension aux relations de quelques propriétés des ordres, Ann. Sci. École Norm. Sup. (4) 71 (1954), 361-388.

2. C. W. Henson, A family of countable homogeneous graphs, Pacific J. Math. 38 (1971), 69-83.

3. Countable homogeneous relational structures and categorical theories, J. Symbolic Logic 37 (1972), 494-500.

4. B. Jonsson, Universal relational systems, Math. Scand. 4 (1956), 193-208.

5. __ Homogeneous universal relational systems, Math. Scand. 8 (1960), 137-142.

6. A. H. Lachlan, Countable ultrahomogeneous tournaments, Abstracts Amer. Math. Soc. 1 (1980), $80 \mathrm{~T}-\mathrm{A} 17$.

7. A. H. Lachlan and R. E. Woodrow, Countable ultrahomogeneous graphs, Trans. Amer. Math. Soc. 262 (1980), 51-94.

8. M. Morley and R. Vaught, Homogeneous universal models, Math. Scand. 11 (1962), 37-52.

9. J. Schmerl, Homogeneous partially ordered sets, preprint.

10. R. E. Woodrow, Theories with a finite number of countable models and a small language, Ph.D. Thesis, Simon Fraser Univ., Burnaby, British Columbia, Canada, 1976.

Department of Mathematics, Simon Fraser University, Burnaby, British Columbia V5A 1S6, CANADA 